\title{
Predicting the in vivo developmental toxicity of benzo[a]pyrene (BaP) in rats by an in vitro-in silico approach
}

\author{
Danlei Wang $^{1}$ (D) Maartje H. Rietdijk ${ }^{1} \cdot$ Lenny Kamelia $^{1,2} \cdot$ Peter J. Boogaard $^{1,2} \cdot$ Ivonne M. C. M. Rietjens $^{1}$
}

Received: 15 March 2021 / Accepted: 29 July 2021 / Published online: 25 August 2021

(c) The Author(s) 2021

\begin{abstract}
Developmental toxicity testing is an animal-intensive endpoints in toxicity testing and calls for animal-free alternatives. Previous studies showed the applicability of an in vitro-in silico approach for predicting developmental toxicity of a range of compounds, based on data from the mouse embryonic stem cell test (EST) combined with physiologically based kinetic (PBK) modelling facilitated reverse dosimetry. In the current study, the use of this approach for predicting developmental toxicity of polycyclic aromatic hydrocarbons (PAHs) was evaluated, using benzo[a]pyrene (BaP) as a model compound. A rat PBK model of $\mathrm{BaP}$ was developed to simulate the kinetics of its main metabolite 3-hydroxybenzo[a]pyrene (3-OHBaP), shown previously to be responsible for the developmental toxicity of BaP. Comparison to in vivo kinetic data showed that the model adequately predicted $\mathrm{BaP}$ and 3-OHBaP blood concentrations in the rat. Using this $\mathrm{PBK}$ model and reverse dosimetry, a concentration-response curve for 3-OHBaP obtained in the EST was translated into an in vivo dose-response curve for developmental toxicity of $\mathrm{BaP}$ in rats upon single or repeated dose exposure. The predicted half maximal effect doses $\left(\mathrm{ED}_{50}\right)$ amounted to 67 and $45 \mathrm{mg} / \mathrm{kg}$ bw being comparable to the $\mathrm{ED}_{50}$ derived from the in vivo dose-response data reported for $\mathrm{BaP}$ in the literature, of $29 \mathrm{mg} / \mathrm{kg}$ bw. The present study provides a proof of principle of applying this in vitro-in silico approach for evaluating developmental toxicity of $\mathrm{BaP}$ and may provide a promising strategy for predicting the developmental toxicity of related PAHs, without the need for extensive animal testing.
\end{abstract}

Keywords Physiologically based kinetic (PBK) model · Alternatives for animal testing · Quantitative in vitro-in vivo extrapolation (QIVIVE) · Benzo[a]pyrene (BaP)

\section{Introduction}

The Registration, Evaluation, Authorization and Restriction of Chemicals (REACH) legislation requires all chemical substances produced or sold within the European Union (EU) at a volume of $\geq 100$ tonnes/year to be evaluated for developmental toxicity. Developmental toxicity testing is one of the most animal-intensive endpoints in toxicity testing, estimated to require more than $20 \%$ of all animals used for toxicity testing under REACH (Jagt et al. 2004). REACH acknowledges the need for alternative, animal-free

Danlei Wang

danlei.wang@wur.nl

1 Division of Toxicology, Wageningen University and Research, Stippeneng 4, 6708 WE Wageningen, The Netherlands

2 Shell Health, Shell International B.V., Carel van Bylandtlaan 16, 2596 HR The Hague, The Netherlands test methods, contributing to the 3Rs (replacement, reduction, refinement) of use of experimental animals in toxicological risk assessment.

Three in vitro test methods are currently scientifically validated for developmental toxicity testing: the limb bud micro mass (MM), the whole embryo culture (WEC) and the mouse embryonic stem cell test (EST) (Genschow et al. 2002, 2004). Only the EST is considered animal-free, as it makes use of the mouse embryonic stem cell line D3 (ESD3) (Buesen et al. 2009). The differentiation assay of the EST evaluates the effect of a compound on the differentiation of ES-D3 cells into beating cardiomyocytes.

However, use of in vitro assays like the EST generates in vitro concentration-response curves, while for toxicological risk assessment, dose-response curves are needed since they enable definition of so-called points of departure (PoDs) to define health-based guidance values for safe human exposure. In vitro concentration-response curves can be translated into in vivo dose-response curves using physiologically 
based kinetic (PBK) modelling-based reverse dosimetry. This approach was previously shown to adequately predict in vivo developmental toxicity of various compounds, using concentration-response data from the EST (Li et al. 2017a; Louisse et al. 2010; Strikwold et al. 2017). The validity of this in vitro-in silico method for polycyclic aromatic hydrocarbons (PAHs) was not yet investigated. This although PAH-containing substances make up a large group of compounds for which REACH legislation dictates developmental toxicity testing. The aim of the present study was to evaluate the use of this in vitro-in silico approach to predict the developmental toxicity of benzo(a)pyrene $(\mathrm{BaP})$. $\mathrm{BaP}$ was chosen as the model compound for PAHs, because $\mathrm{BaP}$ is well studied, and assumed to induce developmental toxicity in rats (Archibong et al. 2002; Bui et al. 1986; Feuston et al. 1989, 1994, 1996; Feuston and Mackerer 1996; Hood et al. 2000; Wu et al. 2003). Furthermore, in vivo kinetic rat data are available in the literature for $\mathrm{BaP}$ and its metabolite 3-hydroxybenzo[a]pyrene (3-OHBaP) (Marie et al. 2010; Moreau and Bouchard 2015) as well as in vivo dose-response data for reproductive toxicity of $\mathrm{BaP}$ in rats (Archibong et al. 2002; Bui et al. 1986), enabling evaluation of the predictions made by the developed in vitro-in silico approach.

$\mathrm{BaP}$ is well known for its bioactivation to diol epoxide metabolites that lead to DNA damage-induced carcinogenicity. For induction of developmental toxicity, BaP needs bioactivation to 3-OHBaP as shown in previous in vitro EST studies (Kamelia et al. 2020). To facilitate the prediction of tissue concentrations for 3-OHBaP, a sub-model for this metabolite was included in the PBK model. Previously, PBK models for $\mathrm{BaP}$ and 3-OHBaP have been developed (Campbell et al. 2016; Crowell et al. 2011; Heredia-Ortiz and Bouchard 2013; Heredia-Ortiz et al. 2011; Heredia Ortiz et al. 2014). However, these models were not applied for reverse dosimetry, leaving the question whether PBK modelling-based reverse dosimetry is suited to predict in vivo developmental toxicity of $\mathrm{BaP}$.

To answer this question, in the present study a PBK model of $\mathrm{BaP}$ in rat was developed for predicting blood concentrations of 3-OHBaP. The model was used to translate concentration-response data for 3-OHBaP from the EST to predict an in vivo dose-response curve for developmental toxicity of $\mathrm{BaP}$ in rats and results obtained were compared to available data in the literature on kinetics and developmental toxicity of $\mathrm{BaP}$ and 3-OHBaP.

\section{Materials and methods}

\section{Materials}

3-OHBaP was ordered from Toronto Research Chemicals (TRC) Canada (North York, Canada). 3'-phosphate 5'-phosphosulfate (PAPS) lithium salt was purchased from Santa
Cruz Biotechnology (Dallas, Texas, United States), BaP, nicotinamide adenine dinucleotide phosphate (NADPH), sodium salt, sodium phosphate, sodium chloride and Trizma ${ }^{\circledR}$ base (TRIS) were purchased at Sigma-Aldrich (Zwijndrecht, The Netherlands). Uridine 5'-diphosphoglucuronic acid (UDPGA) trisodium salt was purchased from Carbosynth (Compton, United Kingdom). Pooled liver and lung S9 fractions and microsomes from male Sprague-Dawley (SD) rats were ordered from Tebu-Bio (Heerhugowaard, The Netherlands). Acetonitrile (ACN) was purchased from Biosolve (Dieuze, France). Dimethyl sulfoxide (DMSO) was obtained from Acros Organics (Geel, Belgium). Potassium hydrogen phosphate $\left(\mathrm{K}_{2} \mathrm{HPO}_{4}\right)$ and trifluoroacetic acid (TFA) were purchased from Merck (Darmstadt, Germany).

\section{Methods}

The PBK modelling-based reverse dosimetry approach consisted of the following steps: (1) defining a PBK model describing the kinetics of 3-OHBaP, the main metabolite of $\mathrm{BaP}$, in rats, (2) determining kinetic parameter values for metabolism of $\mathrm{BaP}$ and conjugation of 3-OHBaP, (3) evaluation of the PBK model using in vivo kinetic literature data, (4) translation of in vitro concentration-response data for 3-OHBaP in the EST (Kamelia et al. 2020) into in vivo dose-response data for developmental toxicity of $\mathrm{BaP}$ using PBK model-facilitated reverse dosimetry, (5) evaluation of the predicted dose-response curve by comparison to the literature reported dose-response data (Archibong et al. 2002; Bui et al. 1986).

\section{Development of a PBK model for BaP and 3-OHBaP in rats}

The PBK model was defined based on the conceptual model for $\mathrm{BaP}$ with a sub-model for 3-OHBaP taking into account the model codes for BaP PBK models that included submodels for 3-OHBaP reported in the literature (Campbell et al. 2016; Crowell et al. 2011; Heredia-Ortiz and Bouchard 2013) and is presented in Fig. 1. The conceptual PBK model for $\mathrm{BaP}$ consisted of separate compartments for venous blood, arterial blood, fat tissue, liver tissue, lung tissue, rapidly and slowly perfused tissue, stomach and intestines. $3-\mathrm{OHBaP}$ is highly lipophilic. To prevent $3-\mathrm{OHBaP}$ from partitioning into the fat tissue in the model simulations, it was essential to include a blood protein compartment in the model to allow binding of 3-OHBaP to blood protein. The unbound fraction of 3-OHBaP in the blood compartment was represented by the $f_{\mathrm{ub} \text {, in vivo, }}$, calculated as described in "Translating in vitro concentration-response data into in vivo dose-response data using PBK modelling-based reverse dosimetry" section. The fraction of 3-OHBaP bound 


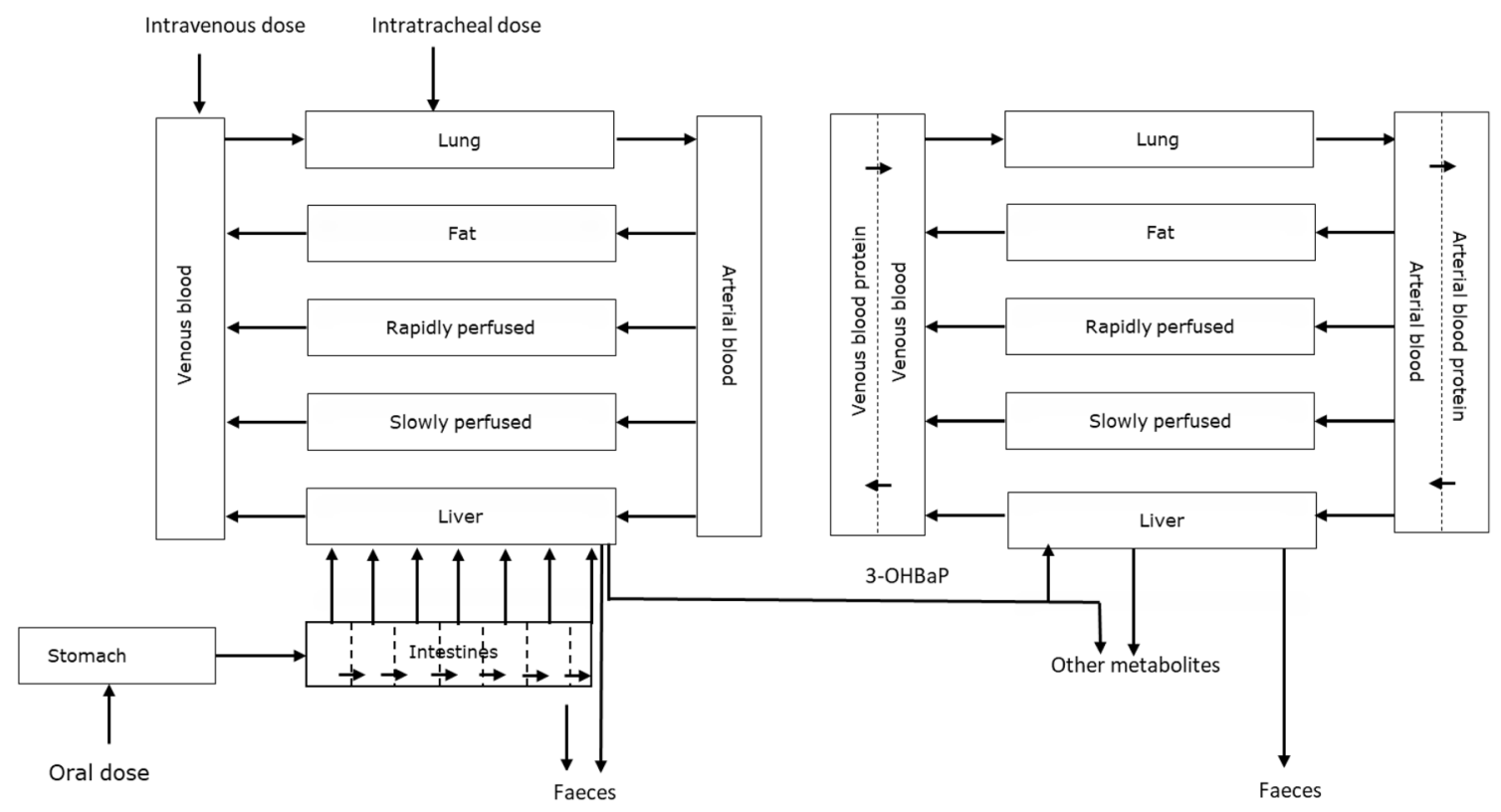

Fig. 1 Schematic overview of the PBK model of BaP containing a sub-model for 3-OHBaP in rat (color figure online)

to protein in the blood protein compartment $\left(f_{\mathrm{b} \text {, in vivo }}\right)$ was

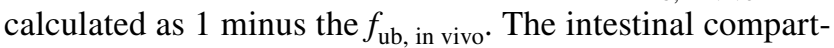
ment was divided into seven sub-compartments to describe the passage of $\mathrm{BaP}$ trough the intestines upon oral exposure (Zhang et al. 2018). Conversion of BaP into 3-OHBaP and other metabolites was initially assumed to occur in the liver and lung (Heredia-Ortiz et al. 2011). Clearance of 3-OHBaP was assumed to result from hepatic and pulmonary conjugation (Cohen 1990; Cohen and Moore 1976). The relative contribution of pulmonary metabolism of $\mathrm{BaP}$ and 3-OHBaP compared to hepatic metabolism of these compounds was investigated as well. Pulmonary metabolism of BaP and 3-OHBaP quantified based on incubations of $\mathrm{BaP}$ with rat lung microsomes and 3-OHBaP with rat lung $\mathrm{S} 9$, calculated as described in "Determining kinetic parameter values for metabolism of $\mathrm{BaP}$ and conjugation of $3-\mathrm{OHBaP}$ " section, was shown to be negligible as compared to metabolism in the liver (see "Development of a PBK model describing the kinetics of $\mathrm{BaP}$ and 3-OHBaP in rats" section) and thus not included in the PBK model. In the model, it is assumed that both $\mathrm{BaP}$ and 3-OHBaP are eliminated to the faeces by biliary excretion.

BaP needs bioactivation to 3-OHBaP to induce developmental toxicity in vitro (Kamelia et al. 2020). Therefore, the PBK model contained a sub-compartment describing the kinetics of 3-OHBaP, enabling prediction of blood concentrations of 3-OHBaP as a function of the dose of $\mathrm{BaP}$, required for the reverse dosimetry. Based on the conceptual model the differential equations were defined and inserted in Berkley Madonna 8.3.18 (UC Berkeley, California, USA) using the Rosenbrock's algorithm for stiff systems. Model equations are included in supplementary materials 1 .

The 3-OHBaP sub-model consisted of the same compartments as the $\mathrm{BaP}$ model, except for stomach and intestine, which were not relevant for the kinetics of 3-OHBaP, since 3-OHBaP is formed in the liver. Studies in pregnant rats have shown that the blood concentration of $\mathrm{BaP}$ in maternal and foetal blood are similar (Withey et al. 1993). The same was found for the concentration of BaP metabolites in maternal and foetal blood (Withey et al. 1993). It was, therefore, assumed that $\mathrm{BaP}$ and its metabolites readily cross the placenta and that the reproductive toxicity observed in vivo is dependent on the maternal blood concentration of 3-OHBaP. For this reason, separate compartments for placental and foetal tissue were not included in the model.

Physiological and anatomical parameter values were taken from literature (Brown et al. 1997; Crowell et al. 2011) and are presented in Table 1.

The tissue:blood partition coefficients for $\mathrm{BaP}$ and 3-OHBaP were previously calculated by Crowell et al. (2011), according to the method of Poulin and Krishnan (1995) and Poulin and Theil (2000) and were applied in the current PBK model (Table 1).

Given the nature of the in vivo data available for model evaluation, and evaluation of the predicted toxicity, the model included single and repeated intravenous, intratracheal and oral exposure to $\mathrm{BaP}$. For oral exposure, stomach emptying and intestinal transfer of the parent compound were included. The uptake of $\mathrm{BaP}$ from the intestines to the liver was described for the seven sub-compartments using the 
Table 1 Physiological, anatomical and physicochemical parameter values for $\mathrm{BaP}$ and 3-OHBaP for the rat PBK model

\begin{tabular}{|c|c|c|c|}
\hline Model parameter & Symbol & Value & References \\
\hline \multicolumn{4}{|l|}{ Physiological parameters } \\
\hline Body weight & BW & $0.245^{\mathrm{a}}$ & $\begin{array}{l}\text { Marie et al. (2010) } \\
\text { Moreau and Bouchard (2015) }\end{array}$ \\
\hline \multicolumn{4}{|l|}{ Fractional tissue volumes } \\
\hline Fat & $\mathrm{VFc}$ & 0.065 & Crowell et al (2011), based on Brown et al. (1997) \\
\hline Liver & VLc & 0.037 & \\
\hline Lung & VLuc & 0.005 & \\
\hline Arterial blood & $\mathrm{VABc}$ & 0.0257 & \\
\hline Venous blood & $\mathrm{VVBc}$ & 0.0514 & \\
\hline Rapidly perfused tissue & VRc & 0.2159 & \\
\hline Slowly perfused tissue & VSc & 0.6 & \\
\hline Cardiac output (mL/s) & QC & $15 * \mathrm{BW}^{0.74}$ & \\
\hline \multicolumn{4}{|l|}{ Fractional tissue blood flows } \\
\hline Fat & QFc & 0.07 & \\
\hline Liver & QLc & 0.183 & \\
\hline Lung & QLuc & 1 & \\
\hline Rapidly perfused tissue & QRc & 0.4 & \\
\hline Slowly perfused tissue & QSc & 0.347 & \\
\hline \multicolumn{4}{|l|}{ Physicochemical parameters } \\
\hline \multicolumn{4}{|l|}{ Benzo[a]pyrene } \\
\hline Molecular weight & MWBaP & 252.31 & \\
\hline $\log P$ & & 6.0 & \\
\hline Fraction unbound & $f_{\mathrm{ub}}$ & 0.006 & Calculated according to Lobell and Sivarajah (2003) \\
\hline \multicolumn{4}{|c|}{ Tissue:blood partition coefficients } \\
\hline Fat & PFBAP & 496.38 & Crowell et al. (2011) based on Poulin and Theil (2000) \\
\hline Liver & PLBAP & 13.31 & \\
\hline Lung & PLuBAP & 13.31 & \\
\hline Rapidly perfused tissue & PRBAP & 13.31 & \\
\hline Slowly perfused tissue & PSBAP & 6.99 & \\
\hline \multicolumn{4}{|l|}{ 3-hydroxybenzo[a]pyrene } \\
\hline Molecular weight & MW3OHBaP & 268.3 & \\
\hline $\log P$ & & 5.9 & \\
\hline Fraction unbound & $f_{\mathrm{ub}}$ & 0.007 & Calculated according to Lobell and Sivarajah (2003) \\
\hline \multicolumn{4}{|c|}{ Tissue:blood partition coefficients } \\
\hline Fat & PF3OHBAP & 401 & Crowell et al. (2011) based on Poulin and Krishnan (1995) \\
\hline Liver & PL3OHBAP & 12.24 & \\
\hline Lung & PLu3OHBAP & 12.24 & \\
\hline Rapidly perfused tissue & PR3OHBAP & 12.24 & \\
\hline Slowly perfused tissue & P3OH BAP & 6.43 & \\
\hline
\end{tabular}

${ }^{a}$ Median of body weights of rats in these two studies

apparent in vivo permeability coefficient $\left(P_{\text {app, in vivo }}\right)$ value. The $P_{\text {app, in vivo }}$ was derived from the in vitro $P_{\text {app, Caco-2 }}$ value for $\mathrm{BaP}$ that was previously determined in the Caco-2 model (Goth-Goldstein et al. 1999). The $P_{\text {app, in vivo was calculated }}$ using the following equation: $\log \left(P_{\text {app, in vivo }}\right)=0.6836 \times \log$ $\left(P_{\text {app, Caco-2 }}\right)-0.5579$ (Sun et al. 2002) and applied in the model as described before (Zhang et al. 2018).
Hepatic conversion of $\mathrm{BaP}$ into 3-OHBaP and other metabolites was described by the $V_{\max }$ and $K_{\mathrm{m}}$, determined in vitro using incubations with rat liver microsomes. Pulmonary metabolism of BaP was shown to be not relevant for the PBK model based on results from incubations with rat lung microsomes and $\mathrm{BaP}$ as described in "Determining kinetic parameter values for metabolism of $\mathrm{BaP}$ and conjugation of 3-OHBaP" section. 
Clearance of 3-OHBaP was assumed to be the result of conjugation in liver tissue. Sulfation and glucuronidation are the main contributors to 3-OHBaP clearance (Cohen 1990; Cohen and Moore 1976). Based on the results of incubation experiments described in "Determining kinetic parameter values for metabolism of $\mathrm{BaP}$ and conjugation of 3-OHBaP" section, pulmonary conjugation was considered irrelevant for clearance of 3-OHBaP in the PBK model. $V_{\max }$ and $K_{\mathrm{m}}$ values for glucuronidation and sulfation of $3-\mathrm{OHBaP}$ in rat liver were determined in in vitro incubations with rat liver S9, performed as described in "Determining kinetic parameter values for metabolism of $\mathrm{BaP}$ and conjugation of 3-OHBaP" section.

All in vitro $V_{\max }$ values were scaled to microsomal or S9 protein content of rat liver using the following scaling factors: $45 \mathrm{mg}$ microsomal protein per gram liver tissue and $125 \mathrm{mg} \mathrm{S} 9$ protein per gram liver tissue (Houston and Galetin 2008). The scaled $V_{\max }$ values were subsequently converted to $V_{\max }$ in $\mathrm{nmol} / \mathrm{min} /$ liver using the liver weight of $9.1 \mathrm{~g}$, calculated from the body weight and fractional liver weight presented in Table 1 . The $K_{\mathrm{m}}$ in vitro was assumed equal to the $K_{\mathrm{m}}$ in vivo.

\section{Determining kinetic parameter values for metabolism of $\mathrm{BaP}$ and conjugation of 3-OHBaP}

Metabolism of BaP The formation of 3-OHBaP and other metabolites from $\mathrm{BaP}$ in liver and lung tissue was investigated in incubations with rat liver microsomes and rat lung microsomes. The incubation mixtures consisted of (final concentrations) $0.1 \mathrm{mM}$ potassium phosphate $(\mathrm{pH} 7.4)$, $5 \mathrm{mM} \mathrm{MgCl}, 0.5 \mathrm{mg} / \mathrm{ml}$ rat liver microsomes or $2 \mathrm{mg} / \mathrm{ml}$ rat lung microsomes, and $1 \mathrm{mM} \mathrm{NADPH}$ in conical glass vials. Incubation mixtures were pre-incubated for $1 \mathrm{~min}$, after which the reaction was initiated by the addition of $\mathrm{BaP}$ from 100 times concentrated stock solutions in DMSO to reach the final volume of $200 \mu \mathrm{l}(1 \% \mathrm{DMSO} v / \mathrm{v})$ with $\mathrm{BaP}$ concentrations ranging from 0 to $200 \mu \mathrm{M}$. The mixtures were incubated in a shaking water bath at $37{ }^{\circ} \mathrm{C}$ for $30 \mathrm{~min}$. $20 \mu \mathrm{l}$ ice-cold $10 \%(\mathrm{v} / \mathrm{v})$ perchloric acid $\left(\mathrm{HClO}_{4}\right)$ was added to terminate the reactions and the mixtures were put on ice for at least $15 \mathrm{~min}$. Di-isopropyl ether (DIPE) was used to extract $\mathrm{BaP}$ and its metabolites from the incubation mixture. To this end, $1 \mathrm{ml}$ DIPE was added to each incubation mixture, the tubes vortexed for $20 \mathrm{~s}$ and the upper layer was collected. Extraction was performed three times. Remaining DIPE was removed by evaporation under a stream of $\mathrm{N}_{2}$. Subsequently, extracts were re-dissolved in $100 \mu \mathrm{l}$ methanol and transferred to UPLC vials for analysis.

Conjugation of 3-OHBaP Incubations with rat liver and lung S9 fractions were optimized to establish linearity over time 


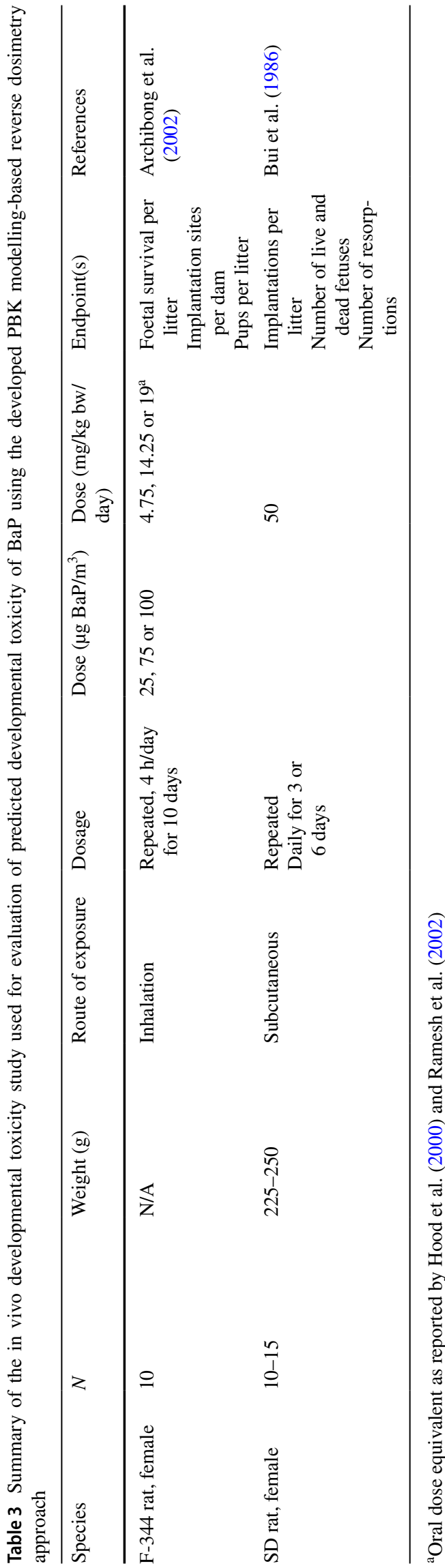

and protein concentrations for the rate of glucuronidation and sulfation of 3-OHBaP. The experiments performed for time optimization revealed that the pulmonary formation rate of sulfated and glucuronidated metabolites of 3-OHBaP was negligible compared to the formation rate of sulfonated and glucuronidated metabolites by the liver (see "Evaluation of the PBK model and sensitivity analysis" section). Pulmonary conjugation was, therefore, considered not relevant for in vivo clearance of 3-OHBaP in the current PBK model and no further experiment for in vitro kinetics with lung fractions were performed.

Kinetics for 3-OHBaP glucuronidation, were quantified using incubations with pooled liver S9 fractions from male SD rats. Incubation mixtures in a final volume of $200 \mu \mathrm{l}$ in conical glass vials consisted of (final concentrations) $0.1 \mathrm{mM}$ Tris- $\mathrm{HCl}$ (pH 7.4), $5 \mathrm{mM} \mathrm{MgCl}, 0.1 \mathrm{mg} / \mathrm{ml}$ rat liver S9, $3 \mathrm{mM}$ UDPGA and $0.025 \mathrm{mg} / \mathrm{ml}$ alamethicin.

Hepatic sulfonation of 3-OHBaP was evaluated using pooled liver S9 fractions of male SD rats, in incubation mixtures with a final volume of $200 \mu \mathrm{l}$ in conical glass vials containing (final concentrations) $0.1 \mathrm{mM}$ Tris- $\mathrm{HCl}$ ( $\mathrm{pH} 7.4$ ), $0.1 \mathrm{mg} / \mathrm{ml}$ rat liver $\mathrm{S} 9$ and $0.2 \mathrm{mM}$ PAPS.

All incubation mixtures were pre-incubated for $1 \mathrm{~min}$, after which the reaction was initiated by addition of $3-\mathrm{OHBaP}$ in final concentrations ranging from 0.01 to $50 \mu \mathrm{M}$ (glucuronidation) or $0.01-100 \mu \mathrm{M}$ (sulfation) added from 100 times concentrated stock solutions in DMSO to reach the final volume of $200 \mu \mathrm{l}(1 \% \mathrm{DMSO} v / \mathrm{v})$. The mixtures were incubated in a shaking water bath at $37^{\circ} \mathrm{C}$ for $20 \mathrm{~min}$ (glucuronidation) or $70 \mathrm{~min}$ (sulfation). $100 \mu \mathrm{l}$ of ice-cold acetonitrile was added to terminate the reactions and the mixtures were put on ice for at least $15 \mathrm{~min}$. Subsequently, the tubes were centrifuged at $4{ }^{\circ} \mathrm{C}$ and $3717 \mathrm{~g}$ per minute for $5 \mathrm{~min}$. The supernatant was collected and analysed by Ultra Performance Liquid Chromatography (UPLC). All incubations were performed in triplicate.

UPLC analysis The collected supernatants were analysed using a UPLC Nexera series (Shimadzu, Kyoto, Japan) to quantify the metabolites of $\mathrm{BaP}$ and the conjugates of 3-OHBaP formed in incubations with rat liver microsomes and S9, respectively. The UPLC was equipped with a Photodiode Array (PDA) detector, recording wavelengths between 190 and $400 \mathrm{~nm}$ and a Phenomenex C18 column (Phenomenex, Torrance, California, United States). The column temperature was kept at $40{ }^{\circ} \mathrm{C}$ and the auto-sampler at $4{ }^{\circ} \mathrm{C}$ during analysis. The mobile phase consisted of Nanopure water containing $0.1 \%(\mathrm{v} / \mathrm{v})$ trifluoroacetic acid (TFA) (A) and acetonitrile containing $0.1 \%(\mathrm{v} / \mathrm{v})$ TFA (B) at a flow rate of $0.3 \mathrm{ml} / \mathrm{min}$. The total run time was $23 \mathrm{~min}$ and $30 \mathrm{~s}$, starting with $10 \%$ B for $30 \mathrm{~s}$, increasing to $100 \% \mathrm{~B}$ in 15 min, maintaining this condition for $3 \mathrm{~min}$ before returning to the initial conditions of $10 \%$ B in $30 \mathrm{~s} .10 \mu \mathrm{l}$ of sample was injected 


$$
\mathbb{I}
$$


per run. Under these conditions the metabolite 3-OHBaP, detected at $258 \mathrm{~nm}$, eluted at $10.16 \mathrm{~min}$. For the glucuronidated and sulfonated metabolite of $3-\mathrm{OHBaP}$, retention time and detection wavelength were $7.5 \mathrm{~min}, 303.3 \mathrm{~nm}$ and $7.4 \mathrm{~min}, 301 \mathrm{~nm}$, respectively. The amounts of $3-\mathrm{OHBaP}$ in the microsomal incubation sample and of glucuronidated and sulfated 3-OHBaP in the S9 incubation samples were quantified by integrating peak areas at their respective wavelengths using a calibration curve prepared with commercially available 3-OHBaP. To obtain the $V_{\max }$ and $K_{\mathrm{m}}$ the in vitro data for the substrate concentration-dependent rate of metabolite formation were fitted to the MichaelisMenten equation using Graphpad Prism 9.0.1 for Windows (GraphPad Software, San Diego, California, USA).

\section{PBK model evaluation and sensitivity analysis}

The PBK model performance was evaluated by comparing predicted time-dependent blood concentrations of $3-\mathrm{OHBaP}$ to reported in vivo time-dependent blood concentrations in rats after dosing $\mathrm{BaP}$ intravenously (Marie et al. 2010; Moreau and Bouchard 2015), intratracheally, and orally (Moreau and Bouchard 2015). Model development and evaluation was focussed on accurate prediction of 3-OHBaP, because the reverse dosimetry is based on the EST data for 3-OHBaP-mediated induction of in vitro developmental toxicity. Further evaluation of the PBK model was done by comparing predicted dose-response data, obtained by reverse dosimetry of the EST data of 3-OHBaP, to in vivo dose-response data of reproductive toxicity of $\mathrm{BaP}$ in rats (Archibong et al. 2002; Bui et al. 1986), performed as described in "Evaluation of predicted dose-dependent developmental toxicity effect" section. Given the nature of the in vivo study used for evaluation of the predicted dose-response curve, the blood concentrations of 3-OHBaP were also predicted for repeated daily exposure to $\mathrm{BaP}$, until steady state of the $C_{\max }$ of $3-\mathrm{OHBaP}$ was reached. An overview of the characteristics of the in vivo kinetic and dose-response studies used for model evaluation is presented in Tables 2 and 3.

For further evaluation of the PBK model, the parameters that were most influential for the prediction of the maximum blood concentration $\left(C_{\max }\right)$ of 3-OHBaP upon intravenous, intratracheal and oral exposure to $\mathrm{BaP}$ were identified by a sensitivity analysis. The sensitivity analysis was performed for intravenous, intratracheal and oral exposure. To this end, each parameter value $(P)$ was increased by $10 \%\left(P^{\prime}\right)$, while keeping the other parameter values constant and the total fraction of arterial and venous blood flow at 1 , resulting in an initial $(\mathrm{C})$ and modified $\left(C^{\prime}\right)$ value of the model prediction for the $C_{\max }$ of 3-OHBaP. Sensitivity coefficients (SC) were calculated using the following equation: $\mathrm{SC}=\left(C^{\prime}-C\right) /$ $\left(P^{\prime}-P\right) \times(P / C)($ Evans and Andersen 2000). The sensitivity analysis was performed for a single dose of $10 \mathrm{mg} / \mathrm{kg} \mathrm{bw}$ $\mathrm{BaP}$, as this dose was applied in the kinetic in vivo studies used for evaluation of the model predicted blood concentrations of 3-OHBaP (Marie et al. 2010; Moreau and Bouchard 2015). The median body weight of the rats in these kinetic in vivo studies was $0.245 \mathrm{~kg}$ and was applied in the model when performing the sensitivity analysis.

\section{Translating in vitro concentration-response data into in vivo dose-response data using PBK modelling-based reverse dosimetry}

The in vitro concentration-response curve obtained for 3-OHBaP in the EST (Kamelia et al. 2020) was translated into a predicted in vivo dose-response curve, using PBK modelling-based reverse dosimetry. The in vivo developmental toxicity response to $\mathrm{BaP}$ is assumed to depend on the $C_{\max }$ of unbound 3-OHBaP in the maternal rat blood ( $C_{\mathrm{ub} \text {, in vivo }}$ ). Therefore, the $C_{\mathrm{ub} \text {, in vivo }}$ was set equal to the concentration of unbound 3-OHBaP in vitro $\left(C_{\mathrm{ub}, \text { in vitro }}\right)$. To correct for differences in $f_{\mathrm{ub}}$ between rat blood $\left(f_{\mathrm{ub}, \text { in vivo }}\right)$ and

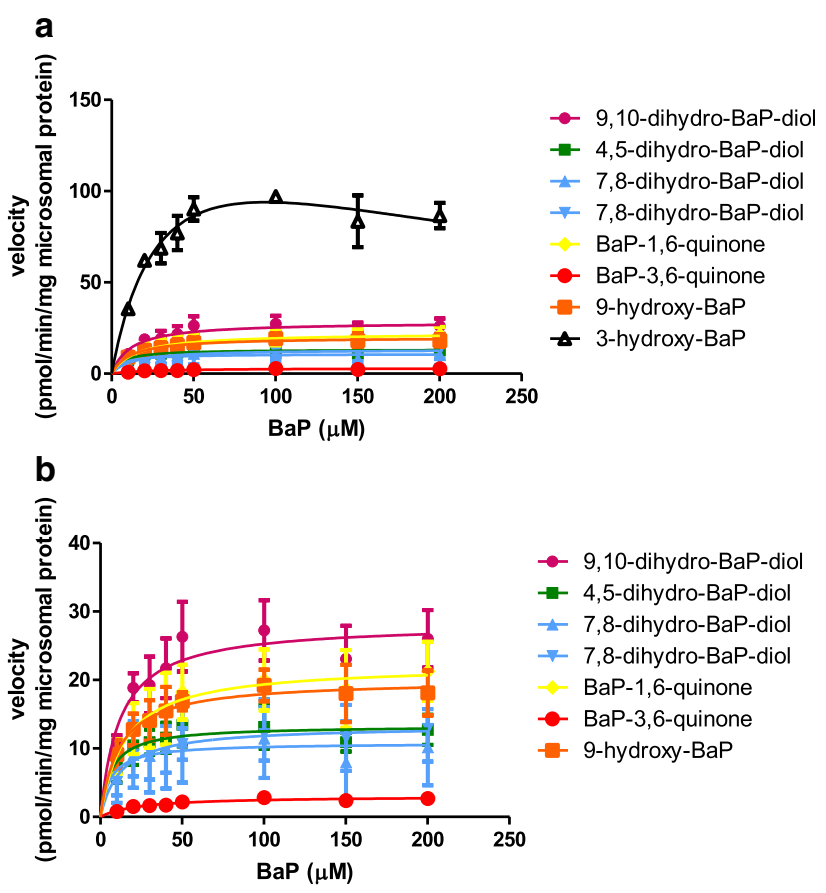

Fig. $2 \mathrm{BaP}$ concentration-dependent formation of $\mathrm{BaP}$ metabolites in incubations with rat liver microsomes (a). Each symbol represents an experimental mean and vertical bars are standard errors of the mean $(n=3)$. Triangle black line, 3-hydroxy-BaP; square orange line, 9-hydroxy-BaP; dot red line, BaP-3,6-quinone; diamond yellow line, $\mathrm{BaP}-1,6$-quinone; both up and down pointing triangle blue line, 7,8-dihydro-BaP-diol (isomers); square green line,4,5-dihydro-BaPdiol; dot pink line, 9,10-dihydro-BaP-diol. To reduce overlap of the respective curves, Fig. $1 \mathrm{~b}$ presents the data for all metabolites except 3-hydroxyBaP on a different $y$-axis scale (color figure online) 
Table 5 Kinetic parameter values for liver metabolism of $\mathrm{BaP}$ and 3-OHBaP in rat

\begin{tabular}{lllll}
\hline & $V_{\max }{ }^{\mathrm{a}}$ & $K_{\mathrm{m}}{ }^{\mathrm{b}}$ & Scaled $V_{\max }{ }^{\mathrm{c}}$ & Scaled $V_{\max }{ }^{\mathrm{d}}$ \\
\hline BaP to 3-OHBaP & 0.16 & 34 & 0.44 & 4.1 \\
BaP to other metabolites & 0.13 & 17 & 0.36 & 3.3 \\
\hline & $V_{\max }{ }^{\mathrm{e}}$ & $K_{\mathrm{m}}(\mu \mathrm{M})^{\mathrm{b}}$ & Scaled $V_{\max }{ }^{\mathrm{c}}$ & Scaled $V_{\max }{ }^{\mathrm{d}}$ \\
\hline Glucuronidated 3-OHBaP & 5.7 & 10 & 43 & 394 \\
Sulfated 3-OHBaP & 0.48 & 17 & 3.6 & 33 \\
\hline
\end{tabular}

${ }^{\mathrm{a}} \mathrm{nmol} / \mathrm{min} / \mathrm{mg}$ microsomal protein

${ }^{\mathrm{b}} \mu \mathrm{M}$

${ }^{\mathrm{c}} \mu \mathrm{mol} / \mathrm{h} / \mathrm{g}$ liver

${ }^{\mathrm{d}} \mu \mathrm{mol} / \mathrm{h} /$ liver

${ }^{\mathrm{e}} \mathrm{nmol} / \mathrm{min} / \mathrm{mg} \mathrm{S} 9$ protein

the EST assay medium ( $\left.f_{\mathrm{ub}, \text { in vitro }}\right)$, the following equation was used:

$$
C_{\text {in vivo }}=\frac{C_{\text {in vitro }} * f_{\mathrm{ub}, \text { in vitro }}}{f_{\mathrm{ub}, \text { in vivo }}}
$$

where $C_{\text {in vivo }}$ is the total 3-OHBaP concentration in the maternal blood, $C_{\text {in vitro }}$ is the total $3-\mathrm{OHBaP}$ concentration used in vitro, $f_{\mathrm{ub} \text {, in vitro }}$ is the fraction unbound in the EST assay medium and $f_{\mathrm{ub} \text {, in vivo }}$ is the fraction unbound in rat blood.

\section{Calculating the fraction unbound $\left(f_{\mathrm{ub}}\right)$ of $3-\mathrm{OHBaP}$ in assay} medium The $f_{\mathrm{ub} \text {, in vivo }}$ of $3-\mathrm{OHBaP}$ was calculated from the $\log P$ value of $3-\mathrm{OHBaP}$ based on the method described previously (Lobell and Sivarajah 2003) using the QIVIVE tool of Wageningen Food Safety Research (WFSR) (https:// wfsr.shinyapps.io/wfsrqivivetools/) (Punt et al. 2021). This in silico method assumes the $f_{\mathrm{ub} \text {, in vivo }}$ in rat plasma for rat to be the same as for human plasma. Furthermore, the $f_{\mathrm{ub} \text {, in vitro }}$ values were assumed to vary linear with the protein content in the biological matrix. This assumption is supported by the linear relationship between the unbound fraction and the albumin concentration in the in vitro test system reported previously for some chlorophenols (Gulden et al. 2002). The fractions bound in vivo $\left(f_{\mathrm{b} \text {, in vivo }}\right)$ were calculated as 1 minus the $f_{\mathrm{ub}, \text { in vivo }}$. The $\log P$ values and calculated $f_{\mathrm{ub} \text {, in vivo }}$ and $f_{\mathrm{b}, \text { in vivo }}$ are presented in Table 1.

The $f_{\mathrm{ub}, \text { in vivo }}$ and $f_{\mathrm{ub} \text {, in vitro }}$ depend on the protein content present in rat blood plasma and assay medium, respectively. The relative amount of protein present in the assay medium used in the EST [15\% (Kamelia et al. 2020)], is approximately twice the protein content of rat blood plasma (7.5\% (Torbert 1935)). Therefore, the $f_{\mathrm{ub} \text {, in vitro }}$ was assumed to be half of the $f_{\mathrm{ub}, \text { in vivo }}$.

PBK modelling-based reverse dosimetry Reverse dosimetry was performed to calculate the dose of $\mathrm{BaP}$ that would give rise to the $C_{\text {in vivo }}$ of 3-OHBaP obtained by setting the in vitro unbound concentrations applied in the EST equal to the unbound in vivo concentration as described in "Translating in vitro concentration-response data into in vivo dose-response data using PBK modelling-based reverse dosimetry" section. Reverse dosimetry was performed for exposure to a single oral dose of $\mathrm{BaP}$ and for repeated daily intravenous and oral dosing. For repeated exposure, $\mathrm{BaP}$ was dosed daily until steady state of the 3-OHBaP blood concentration was reached. BaP doses were calculated using a parameter plot where the maximum blood concentration $\left(C_{\text {in vivo }}\right)$ of 3-OHBaP was plotted against the oral dose of $\mathrm{BaP}(\mathrm{mg} / \mathrm{kg}$ bw). In vivo dose-response data used to evaluate the model predicted dose-response curve were available from literature (Archibong et al. 2002; Bui et al. 1986), the details of these studies are summarized in Table 3.

\section{Evaluation of predicted dose-dependent developmental toxicity}

Conversion of the exposure concentration of $\mathrm{BaP}$ in air to an oral equivalent dose per kg bw The dose-response data predicted from the concentration-response curves derived in the EST (Kamelia et al. 2020) were compared to in vivo dose-response data for reproductive toxicity of $\mathrm{BaP}$ upon nasal inhalation (Archibong et al. 2002) and to the in vivo data for reproductive toxicity of BaP upon subcutaneous injection (Bui et al. 1986). The details of these studies are summarized in Table 3. Foetal survival, calculated as the fraction of live foetuses relative to the number of implantation sites reported in these studies, was taken as measure for in vivo reproductive toxicity. Archibong et al. (2002) exposed Fisher 344 (F-344) rats to 25, 75 or $100 \mu \mathrm{g} \mathrm{BaP} /$ $\mathrm{m}^{3}$ via nasal inhalation, for $4 \mathrm{~h}$ per day, from gestation day 11-20. Previous studies reported that the inhalation doses of 25,75 or $100 \mu \mathrm{g} \mathrm{BaP} / \mathrm{m}^{3}$ correspond to an equivalent oral 
Fig. 3 Concentration-dependent oxidation of $\mathrm{BaP}$ to (a) 3-OHBaP and (b) sum of other metabolites in incubation with rat liver microsomes. Symbols represent the mean of three independent experiments, the error bars represent the standard error of the mean (SEM)
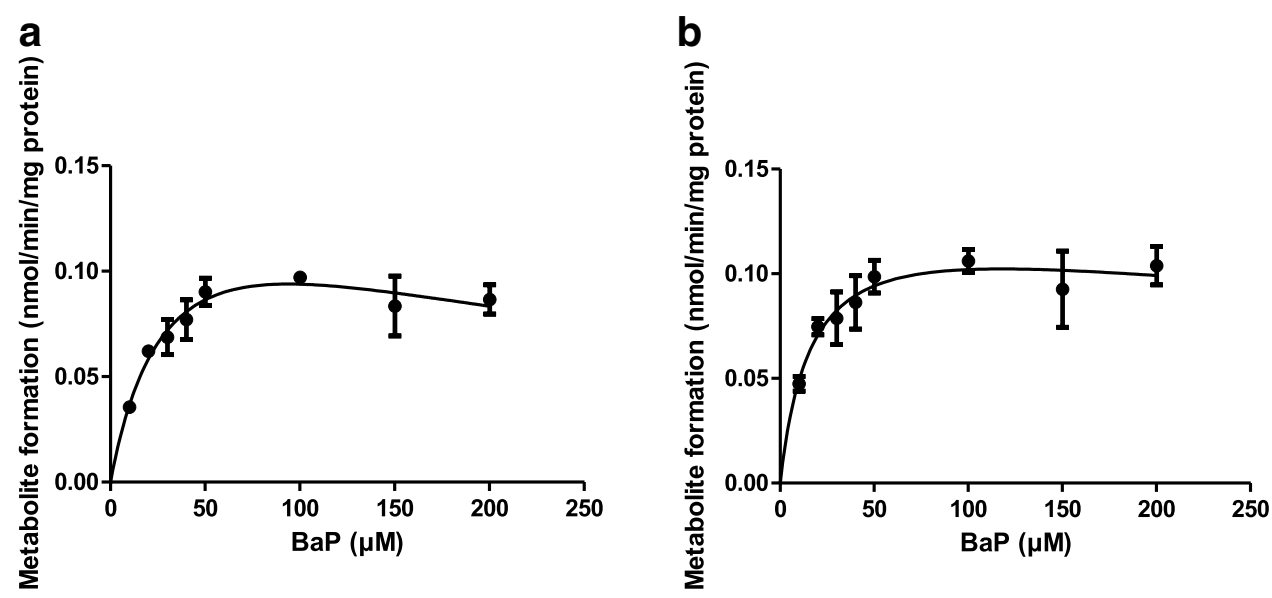

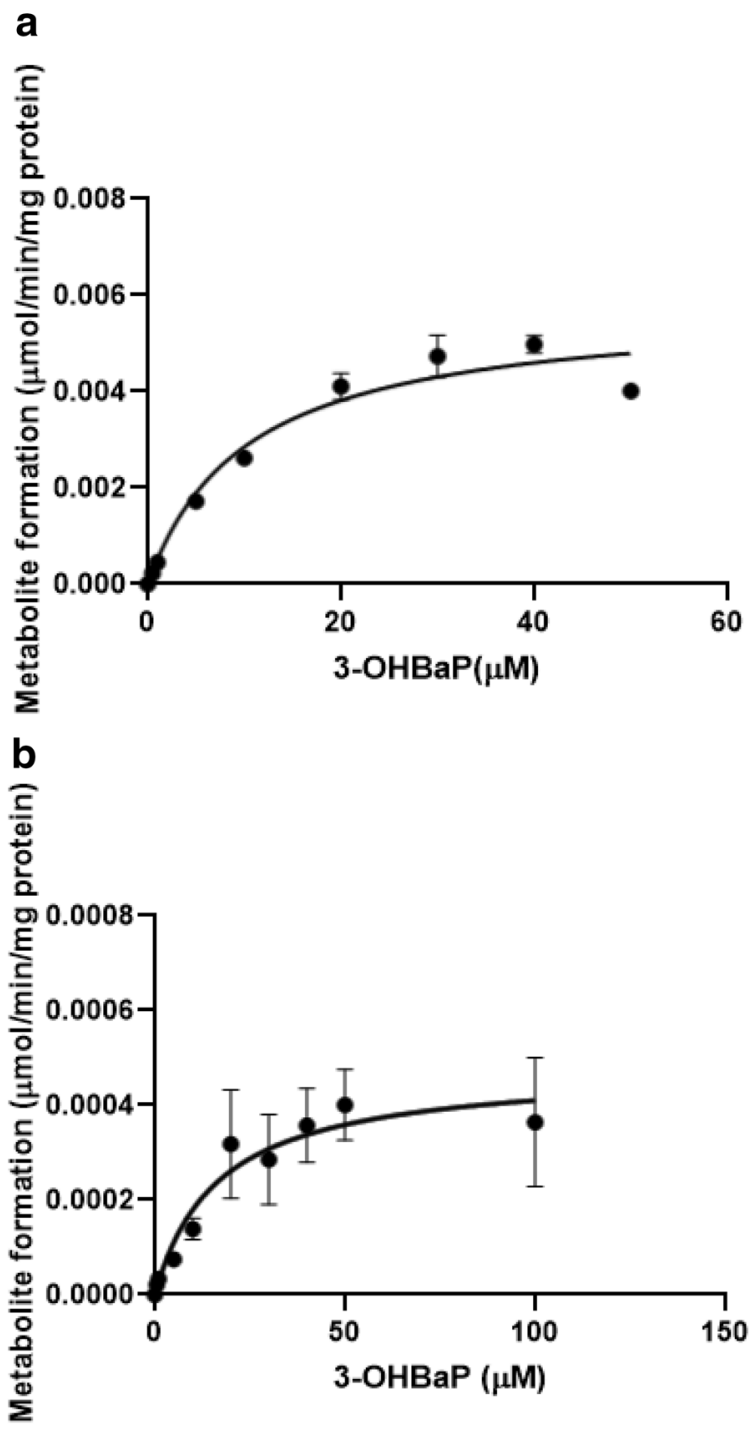

Fig. 4 Concentration-dependent glucuronidation (a) and sulfation (b) of 3-OHBaP in incubations with rat liver S9. Symbols represent the mean of three independent experiments, the error bars represent the standard error of the mean (SEM) dose of 4.75, 14.25 and $19 \mathrm{mg} / \mathrm{kg}$ bw, respectively (Hood et al. 2000; Ramesh et al. 2002). These oral dose equivalents in $\mathrm{mg} / \mathrm{kg}$ bw were used for the comparison with model predicted dose-response data. Bui et al. (1986) exposed pregnant $\mathrm{SD}$ rats to $50 \mathrm{mg} \mathrm{BaP} / \mathrm{kg}$ bw per day via subcutaneous injection from gestation day 6-8 or 6-11.

Calculating the $\mathrm{ED}_{50}$ for evaluating the predicted doseresponse data $\mathrm{ED}_{50}$ values were calculated for the predicted and reported dose-response data, using the non-linear regression with three parameters in Graphpad Prism version 9.0.1 for Windows (GraphPad Software, San Diego, California USA). The $\mathrm{ED}_{50}$ value was calculated for the fraction differentiated into beating cardiomyocytes for the predicted dose-response data. For the in vivo studies, foetus survival (number of live foetuses as fraction of total implantations) was used as response.

\section{Results}

\section{Development of a PBK model describing the kinetics of $\mathrm{BaP}$ and 3-OHBaP in rats}

\section{Kinetics of $\mathrm{BaP}$ and 3-OHBaP in rats}

Figure 2 presents the BaP concentration-dependent formation of metabolites in incubations with rat liver microsomes. Table 4 shows the kinetic parameter values, $V_{\max }, K_{\mathrm{m}}$ and catalytic efficiency that were derived from these data. The metabolites of $\mathrm{B}[\mathrm{a}] \mathrm{P}$ were characterized based on the elution order and reference UV spectra reported in the literature (Chou 1983; Hamernik 1984; Hamernik et al. 1983; Koehl et al. 1996; Moserova et al. 2009; Veignie et al. 2002; Yang et al. 1975) and commercially available reference chemicals. Three cis-B[a]P dihydrodiols were identified as B[a]P-9,10-dihydrodiol, B[a]P-4,5-dihydrodiol and 
Fig. 5 Reported and predicted blood concentrations of $\mathrm{BaP}$ in rats upon (a) intravenous, (b) intratracheal and (c) oral exposure to $10 \mathrm{mg} /$ $\mathrm{kg} \mathrm{BaP}$ in rats. Symbols represent (a) the average blood concentrations reported in the in vivo studies of Moreau and Bouchard (2015) (filled diamonds) and Marie et al. (2010) (open circles) for intravenous exposure, (b) the average blood concentrations reported in the in vivo study of Moreau and Bouchard (2015) (grey open diamonds) and scaled Moreau and Bouchard data based on the fold difference observed in intravenous data (grey open triangles) upon intratracheal exposure, and (c) the average blood concentrations reported in the vivo study of Moreau and Bouchard (2015) (black open diamonds) and scaled Moreau and Bouchard data based on the fold difference observed in intravenous data (black open triangles) upon oral exposure. The lines represent the model predicted blood concentrations upon (a) intravenous (black solid line), (b) intratrachael (grey solid line) and (c) oral (black dashed line) exposure

B[a]P-7,8-dihydrodiol with identical UV spectra to those reported previously (Chou 1983; Hamernik 1984; Hamernik et al. 1983). Another B[a]P-dihydrodiol was identified as a geometric isomer being trans-B[a]P-7,8-dihydrodiol due to spectral similarity to that of cis-B[a]P-7,8-dihydrodiol. Two quinones of $\mathrm{B}[\mathrm{a}] \mathrm{P}$ were identified as BaP-1,6-quinone and B[a]P-3,6-quinone with reported identical spectra (Chou 1983; Veignie et al. 2002). 9-Hydroxy-B[a]P was identified according to the reported UV wavelengths of 9-hydroxyB[a]P (Sims 1968). 3-OHB[a]P was identified by co-elution and identical spectra to that of commercially available 3-OHBaP and the spectra reported by Hamernik (1984).

Given these results, in the PBK model, the metabolism of $\mathrm{BaP}$ was described by two Michaelis Menten equations, one to describe the bioactivation of $\mathrm{BaP}$ to 3-OHBaP, and the other to describe the combined conversion to all other metabolites together. Figure 3 presents the corresponding curves and Table 5 shows the kinetic parameter values, $V_{\max }$ and $K_{\mathrm{m}}$, derived from these data. The $V_{\max }$ of 3-OHBaP formation and the $V_{\max }$ for the sum of formation of all other minor metabolites were 0.16 and $0.13 \mathrm{nmol} / \mathrm{min} / \mathrm{mg}$ microsomal protein, respectively, amounting to 4.1 and $3.3 \mu \mathrm{mol} /$ $\mathrm{min} /$ liver, when scaled to the whole liver using the scaling factor described in "Determining kinetic parameter values for metabolism of $\mathrm{BaP}$ and conjugation of 3-OHBaP" section.

Figure 4 shows the concentration-dependent rate of (a) glucuronidation and (b) sulfation of 3-OHBaP in incubations with rat liver S9. Hepatic glucuronidation and sulfation of 3-OHBaP followed Michaelis-Menten kinetics. Substrate inhibition occurred at concentrations of $50 \mu \mathrm{M}$ and higher for glucuronidation. Table 5 shows the kinetic parameter values, $V_{\max }$ and $K_{\mathrm{m}}$, derived from these data. Incubations of $\mathrm{BaP}$ with lung microsomes did not result in formation of detectable levels of 3-OHBaP, except for the incubations with the highest concentration of $\mathrm{BaP}(200 \mu \mathrm{M})$. The rate of 3-OHBaP formation at $200 \mu \mathrm{M} \mathrm{BaP}$ in rat lung microsomes amounted to $0.81 \mathrm{pmol} / \mathrm{min} / \mathrm{mg}$ protein converted
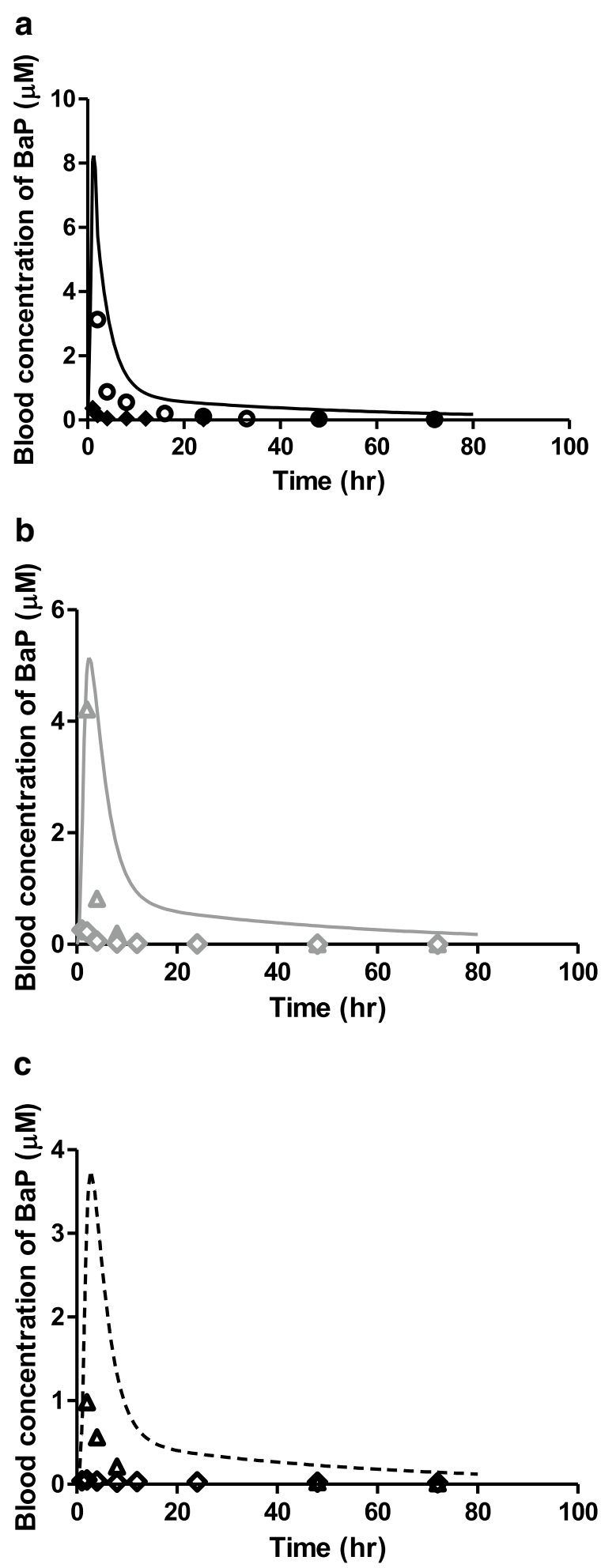

with a scaling factor of $3.67 \mathrm{mg}$ microsomal protein/g lung and a lung weight of $1.25 \mathrm{~g}$ (Table 1) to a rate of conversion of $3.7 \mathrm{pmol} / \mathrm{min} / \mathrm{lung}$ amounting to $0.01 \%$ of the rate of conversion at $200 \mu \mathrm{M} \mathrm{BaP}$ in the liver of $86.6 \mathrm{pmol} / \mathrm{min} /$ $\mathrm{mg}$ microsomal protein, amounting to a rate of conversion of $36.0 \times 10^{3} \mathrm{pmol} / \mathrm{min} /$ liver using the scaling factors for 
Fig. 6 Reported and predicted blood concentrations of 3-OHBaP in rats upon (a) intravenous, (b) intratracheal and (c) oral exposure to $10 \mathrm{mg} / \mathrm{kg} \mathrm{BaP}$ in rats. Symbols represent (a) the average blood concentrations reported in the in vivo studies of Moreau and Bouchard (2015) for intravenous (filled diamonds) and Marie et al. (2010) (open circles) for intravenous exposure. Symbols represent (b) the average blood concentrations reported in the in vivo studies of Moreau and Bouchard (2015) (grey open diamonds) and scaled Moreau and Bouchard data based on the fold difference observed in intravenous data (grey open triangles) upon intratracheal exposure. Symbols represent (c) the average blood concentrations reported in in vivo studies of Moreau and Bouchard (2015) (black open diamonds) and scaled Moreau and Bouchard data based on the fold difference observed in intravenous data (black open triangles) upon oral exposure. The lines represent the model predicted blood concentrations upon (a) intravenous (black solid line), (b) intratracheal (grey solid line) and (c) oral (black dashed line) exposure

microsomal protein content of the liver and liver weight mentioned in "Determining kinetic parameter values for metabolism of $\mathrm{BaP}$ and conjugation of 3-OHBaP" section. Based on this result, it was concluded that 3-OHBaP formation from $\mathrm{BaP}$ in the lung does not add substantially to the overall 3-OHBaP formation and is not to be included in the PBK model.

Incubations of 3-OHBaP with rat lung $\mathrm{S} 9$ and $50 \mu \mathrm{M}$ 3-OHBaP showed that the formation rate of glucuronidated metabolites was $0.015 \mathrm{nmol} / \mathrm{min} / \mathrm{mg} \mathrm{S} 9$ protein, scaled to the whole lung with a scaling factor of $10.19 \mathrm{mg} \mathrm{S} 9$ protein/g lung and a lung weight of $1.25 \mathrm{~g}$ to a conversion rate of $0.19 \mathrm{nmol} / \mathrm{min} / \mathrm{lung}$. For liver, the glucuronidation rate at $50 \mu \mathrm{M} 3-\mathrm{OHBaP}$ was $4.0 \mathrm{nmol} / \mathrm{min} / \mathrm{mg} \mathrm{S} 9$ protein, amounting to $4.6 \times 10^{3} \mathrm{nmol} / \mathrm{min} /$ liver, scaled to the whole liver using the scaling factor described in "Determining kinetic parameter values for metabolism of $\mathrm{BaP}$ and conjugation of 3 -OHBaP" section. This implies that at $50 \mu \mathrm{M} 3-\mathrm{OHBaP}$ the pulmonary conversion rate amounted to approximately $0.04 \%$ of the hepatic conversion rate. For sulfonation, the estimated conversion rate in incubations with rat lung $\mathrm{S} 9$ and $50 \mu \mathrm{M} 3-\mathrm{OHBaP}$ was $0.001 \mathrm{nmol} / \mathrm{min} / \mathrm{mg} \mathrm{S} 9$ protein, amounting to $0.013 \mathrm{nmol} / \mathrm{min} /$ lung using the scaling factors mentioned above. For liver, the sulfation rate of 3-OHBaP at $50 \mu \mathrm{M} 3-\mathrm{OH}-\mathrm{BaP}$ was $0.40 \mathrm{nmol} / \mathrm{min} / \mathrm{mg}$ protein, scaled to the whole liver applying the scaling factors mentioned in "Determining kinetic parameter values for metabolism of $\mathrm{BaP}$ and conjugation of 3-OHBaP" section, resulting in $464 \mathrm{nmol} / \mathrm{min} /$ liver. Thus, for sulfonation, the pulmonary conversion rate is $<0.003 \%$ of the hepatic conversion rate. Based on these results, it was concluded that pulmonary conjugation of 3-OHBaP was negligible and, therefore, did not need to be included in the PBK model.

\section{Evaluation of the PBK model and sensitivity analysis}

Given that 3-OHBaP induces developmental toxicity in the EST (Kamelia et al. 2020) and that the aim of the present
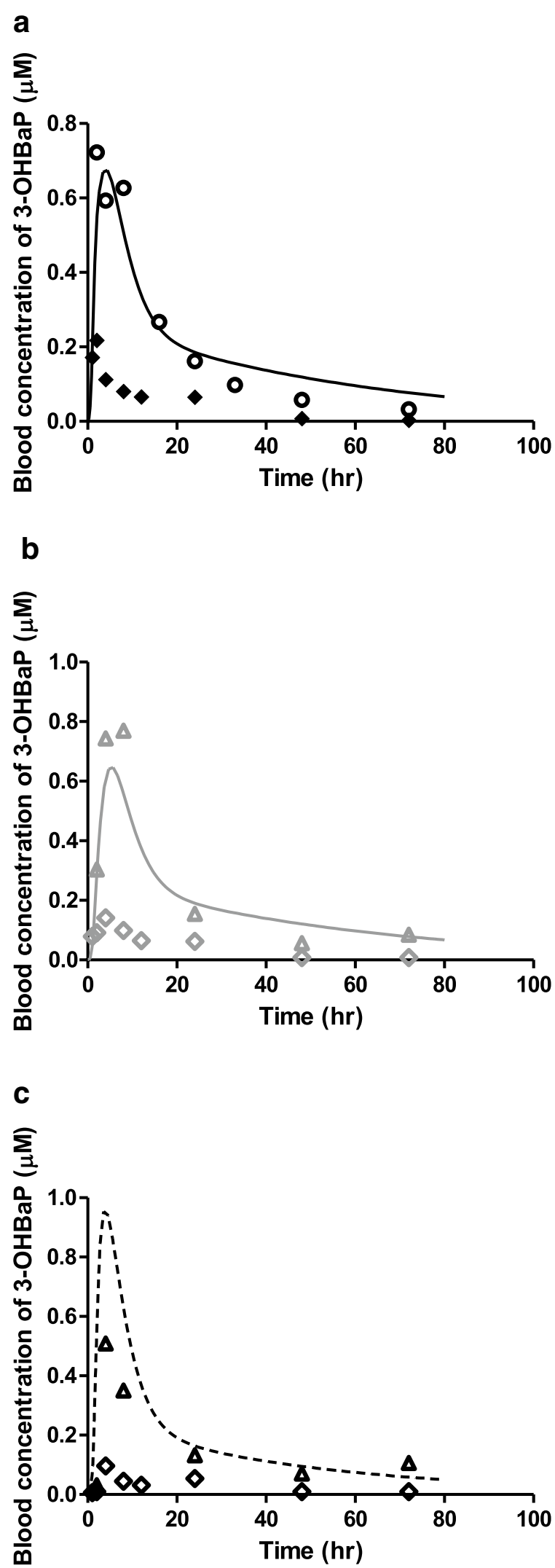
Table 6 Maximum blood concentration $\left(C_{\max }\right)$ of 3-OHBaP in rat blood reported in vivo and predicted by the PBK model upon intravenous, intratracheal and oral administration of $10 \mathrm{mg} / \mathrm{kg}$ bw $\mathrm{BaP}$

\begin{tabular}{lllll}
\hline References & Route of exposure & $\begin{array}{l}C_{\max } \text { pre- } \\
\text { dicted }(\mu \mathrm{M})\end{array}$ & $\begin{array}{l}C_{\max } \\
\text { reported } \\
(\mu \mathrm{M})\end{array}$ & $\begin{array}{l}C_{\max } \\
\text { predicted/C } \\
\text { reported }\end{array}$ \\
\hline Moreau and Bouchard (2015) & Intravenous & 0.68 & 0.22 & 3.1 \\
Marie et al. (2010) & Intravenous & 0.68 & 0.72 & 0.9 \\
Moreau and Bouchard (2015) & Intratracheal & 0.65 & 0.14 & 4.6 \\
Scaled Moreauand Bouchard (2015) & Intratracheal & 0.65 & 0.77 & 0.8 \\
Moreau and Bouchard (2015) & Oral & 0.96 & 0.10 & 9.6 \\
Scaled Moreau and Bouchard (2015) & Oral & 0.96 & 0.51 & 1.9 \\
\hline
\end{tabular}

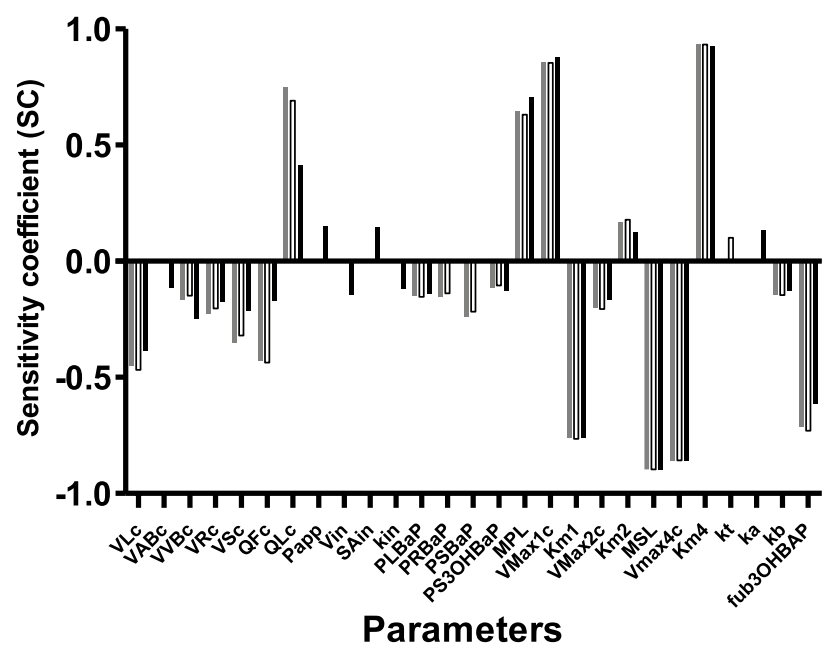

Fig. 7 Sensitivity coefficients (SC) of PBK model parameters for the predicted $\mathrm{Cmax}$ of $3-\mathrm{OHBaP}$ in rat blood after intravenous (grey bars), oral (black bars) or intratracheal (white bars) administration of $10 \mathrm{mg} / \mathrm{kg}$ bw BaP. Model parameters with an absolute $\mathrm{SC}$ of $\geq 0.1$ are shown. $\mathrm{VLc}=$ fraction of liver tissue, $\mathrm{VABc}=$ fraction of arterial blood, $\mathrm{VVBc}=$ fraction of venous blood, $\mathrm{VRc}=$ fraction of rapidly perfused tissue, $\mathrm{VSc}=$ fraction of slowly perfused tissue, $\mathrm{QFc}=$ fraction of blood flow to fat, $\mathrm{QLc}=$ fraction of blood flow to liver, $\mathrm{QLUc}=$ fraction of blood flow to lung, $P a p p=$ apparent intestinal permeability coefficient in vitro obtained in the Caco-2 model, Vin $=$ volume of each compartment of intestines, SAin $=$ surface area intestinal compartment, $\mathrm{kin}=$ transfer rate to next compartment within the intestines, $\mathrm{PLBaP}=$ liver/blood partition coefficient of $\mathrm{BaP}, \mathrm{PRBaP}=$ rapidly perfused tissue:blood partition coefficient of $\mathrm{BaP}, \mathrm{PSBaP}=$ slowly perfused tissue: blood partition coefficient of $\mathrm{BaP}, \mathrm{PS} 3 \mathrm{OHBaP}=$ slowly perfused tissue:blood partition coefficient of $3-\mathrm{OHBaP}, \mathrm{MPL}=$ microsomal protein content in liver, $V \max 1 \mathrm{c}=$ maximum rate of $3-\mathrm{OHBaP}$ formation in liver, $\mathrm{Km} 1=$ Michaelis - Menten constant for metabolism of $\mathrm{BaP}$ to $3-\mathrm{OHBaP}$ in liver, $\mathrm{Vmax} 2 \mathrm{c}=$ maximum rate formation of other metabolites in liver, $\mathrm{Km} 2=$ Michaelis-Menten constant for metabolism of $\mathrm{BaP}$ to other metabolites, $\mathrm{MSL}=\mathrm{S} 9$ protein content in liver, $\mathrm{Vmax} 4 \mathrm{c}=$ maximum rate of glucuronidation of $3-\mathrm{OHBaP}$ formation in liver, $\mathrm{Km} 4=$ Michaelis - Menten constant for glucoronidation $3-\mathrm{OHBaP}$ in liver, $\mathrm{kt}=$ absorption constant from tracheal to lung of $\mathrm{BaP}, \mathrm{ka}=$ absorption constant stomach of $\mathrm{BaP}$ to GI-tract, $\mathrm{kb}=$ excretion constant bile to faeces of $\mathrm{BaP}$, fubBaP $=$ fraction unbound of $\mathrm{BaP}$, fub3OHBaP$=$ fraction unbound of $3-\mathrm{OHBaP}$ study was to translate in vitro EST data on 3-OHBaP to an in vivo dose-response curve for developmental toxicity of $\mathrm{BaP}$, evaluation of the BaP PBK model focussed on the accuracy of predicting the 3-OHBaP levels. Figure 5 presents the model predictions for $\mathrm{BaP}$ and their comparison to the literature reported values for blood $\mathrm{BaP}$ concentrations upon (a) intravenous, (b) intratracheal and (c) oral exposure to a dose of $10 \mathrm{mg} \mathrm{BaP} / \mathrm{kg}$ bw/day. Figure 6 shows a comparison of the predicted time-dependent blood concentrations of 3-OHBaP to reported in vivo time-dependent blood concentrations of 3-OHBaP in rats upon (a) intravenous, (b) intratracheal and (c) oral exposure to a dose of $10 \mathrm{mg} \mathrm{BaP} / \mathrm{kg}$ bw/day. The data presented in Figs. 5a and $6 \mathrm{a}$ reveal that the model predictions for both $\mathrm{BaP}$ and 3-OHBaP match the experimental data of Marie et al. (2010) well, while the data of Moreau and Bouchard (2015), which were obtained at a similar dose level, report blood concentrations that are substantially lower than those reported by Marie et al. and/or the predictions. Table 6 summarises the maximum blood concentrations $\left(C_{\max }\right)$ of $3-\mathrm{OHBaP}$ in rat blood reported in vivo and predicted by the PBK model. Although in both experimental in vivo studies rats of the same strain were exposed intravenously to the same dose of $10 \mathrm{mg} / \mathrm{kg}$ bw/day, the predicted $C_{\max }$ values differed. As a result, the $C_{\max }$ reported by Marie et al. (2010) was 1.1 fold higher and that of Moreau and Bouchard (2015) was 3.1 fold lower than the predicted $C_{\max }$. The predicted $C_{\max }$ of 3-OHBaP was 4.6 and 9.6 fold higher upon intratracheal and oral exposure to $\mathrm{BaP}$, respectively, compared to the $C_{\max }$ of 3-OHBaP reported in the study of Moreau and Bouchard (2015) (Fig. 6b, c). The study by Marie et al. (2010) did not report data for intratracheal or oral dosing, and as a result, a comparison between predictions and experimental data for these routes of administration could only be made using the data of Moreau and Bouchard. When correcting these experimental data for intratracheal and oral dosing using the factor difference observed between the two experimental data sets upon intravenous dosing, the scaled experimental data of Moreau and Bouchard match the model predictions much better. The predicted $C_{\max }$ of 3-OHBaP was 0.8 and 1.9 fold higher upon intratracheal and oral exposure to 


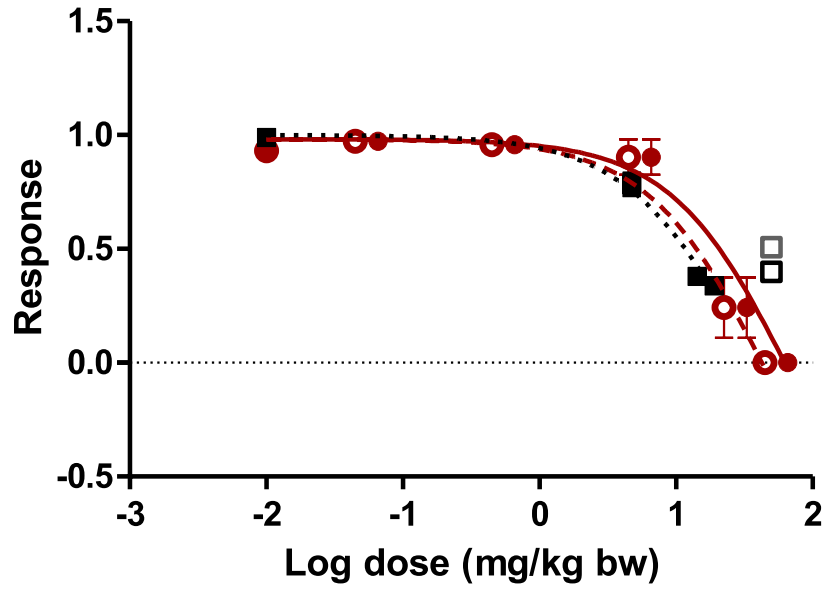

Fig. 8 Model predicted and reported in vivo dose-response curves for developmental toxicity of $\mathrm{BaP}$ in rats upon single and repeated oral exposure. The predicted dose-response curves are translated from the concentration response curve obtained in the EST for 3-OHBaP (Kamelia et al. 2020) representing single oral exposure (filled red circles and red solid line) and repeated oral exposure (red open circles and red dashed line). Filled black squares and dotted black line represent the in vivo data of Archibong et al. (2002), the open squares represent the data of Bui et al. (1986) for 3 day exposure (black open square) and 6 day exposure (grey open square). The error bars represent the standard error of the mean (SEM) (color figure online)

$\mathrm{BaP}$, respectively, compared to the scaled $C_{\max }$ of 3-OHBaP reported in the study of Moreau and Bouchard (2015). The apparent discrepancy between the two available in vivo studies is not surprising considering that the mean value of 3-OHBaP recovery in the Moreau and Bouchard study was reported to be relatively low ( $43 \%$ in blood).

Based on these observations, it was concluded that the PBK model predicted the plasma $C_{\max }$, for 3-OHBaP well enough for further use and evaluation of the PBK model using it for PBK model based reverse dosimetry. The predictions made by the PBK model based reverse dosimetry may then also be used to further evaluate the model and its predictions.

To enable prediction of repeated dose exposures, the PBK model was extended to allow repeated daily dosing. Steady state in the blood concentration of 3-OHBaP upon repeated exposure to $\mathrm{BaP}$ was reached after approximately 15 repetitions for all 3 routes of exposure. The $C_{\max }$ values for 3-OHBaP predicted for repeated intravenous, intratracheal and oral exposure to $\mathrm{BaP}$ were 1.7, 1.7 and 1.4 times higher than the predicted $C_{\max }$ values upon a single $\mathrm{BaP}$ dose for these three routes of exposure. A figure presenting the model predicted time-dependent blood concentrations for repeated intravenous, intratracheal and oral exposure to $\mathrm{BaP}$ is included in supplementary materials 2 (figure $\mathrm{S} 1$ ).

A sensitivity analysis was performed to identify the parameter values that have the highest influence on the model simulations for the $C_{\max }$ of 3-OHBaP in blood upon intratracheal, intravenous and oral exposure to $10 \mathrm{mg} / \mathrm{kg}$ bw $\mathrm{BaP}$. Sensitivity coefficients with an absolute value of 0.1 and higher are shown in Fig. 7. Parameters related to fractional blood flow to liver tissue (QLc), the fraction unbound of 3-OHBaP (fub3OHBaP), microsomal and S9 protein content of the liver (MPL and MSL) and kinetic parameters for the metabolism of $\mathrm{BaP}$ and glucuronidation of 3-OHBaP $\left(K_{\mathrm{m} 1}, K_{\mathrm{m} 4}, V_{\max 1 c}, V_{\max 4 \mathrm{c}}\right)$ were found to be most influential on the simulated $C_{\max }$ of 3-OHBaP.

\section{Translating in vitro concentration-response data into in vivo dose-response data using PBK modelling-based reverse dosimetry}

Using the PBK model thus obtained and evaluated, the in vitro concentration-response data from the EST for 3-OHBaP were translated into a dose-response curve for the developmental toxicity of BaP, after correction for differences in free fraction of 3-OHBaP in vivo and in vitro. Differences in protein binding between the in vitro and in vivo situation where corrected for as described in the materials and methods in "Translating in vitro concentration-response data into in vivo dose-response data using PBK modelling-based reverse dosimetry" section with the values for $f_{\mathrm{ub} \text {, in vitro }}$ of 0.007 and $f_{\mathrm{ub} \text {, in vivo }}$ of 0.0035 . This correction provides the total blood $C_{\max }$ value that matches the total in vitro concentration in the EST corrected for protein binding. Using a curve relating, the PBK model predicted total blood $C_{\max }$ values of 3-OHBaP to the oral dose levels of $\mathrm{BaP}$, the EST concentration-response curve for in vitro developmental toxicity of 3-OHBaP was converted to an in vivo dose-response curve for developmental toxicity of $\mathrm{BaP}$. The predicted dose-response curve for single exposure to $\mathrm{BaP}$ thus obtained is shown in Fig. 8. In addition, Fig. 8 also presents the dose-response curve predicted based on the steady-state $C_{\max }$ values obtained upon repeated oral exposure to $\mathrm{BaP}$.

\section{Evaluation of the PBK modelling-based reverse dosimetry based predictions}

To evaluate the predicted dose-response curves for developmental toxicity of BaP, Fig. 8 also presents the in vivo dose-response data on reproductive toxicity of $\mathrm{BaP}$, represented by the foetal survival, reported in the literature upon exposure of rats to $\mathrm{BaP}$ via nasal inhalation for 10 days (Archibong et al. 2002) and for subcutaneous BaP exposure for 3 and 6 days in rats (Bui et al. 1986). For this comparison, the inhalation dose levels in the study of Archibong et al. (2002) were expressed in equivalent oral dose levels in $\mathrm{mg} / \mathrm{kg}$ bw per day reported previously (Hood et al. 2000; Ramesh et al. 2002) (Table 3). Visual comparison 
of the dose-response curves reveals that the predicted dose-response curves are in agreement with the experimental data. This further evaluates the PBK model for prediction of 3-OHBaP $C_{\max }$ values upon exposure to $\mathrm{BaP}$.

The $\mathrm{ED}_{50}$ values derived from the predicted and reported dose-response curves, amount to $67 \mathrm{mg} / \mathrm{kg}$ bw for single oral exposure to $\mathrm{BaP}, 45 \mathrm{mg} / \mathrm{kg}$ bw per day for repeated oral exposure to $\mathrm{BaP}$ and $29 \mathrm{mg} / \mathrm{kg}$ bw per day for the reported in vivo data (Archibong et al. 2002). From the in vivo data of Bui et al. (1986), no $\mathrm{ED}_{50}$ values could be calculated, but the reported effect doses are in line with the model predictions, corroborating the accuracy of the predicted dose-response curves.

\section{Discussion}

In vivo studies have demonstrated the reproductive toxicity of BaP (Archibong et al. 2002, 2012; Bui et al. 1986). The aim of the present study was to evaluate the use of an in vitro-in silico approach using PBK model-facilitated reverse dosimetry for predicting the developmental toxicity of $\mathrm{BaP}$ based on in vitro toxicity data from the EST for 3-OHBaP, the main metabolite of $\mathrm{BaP}$ responsible for the developmental toxicity of $\mathrm{BaP}$ in the EST (Kamelia et al. 2020). The intermediate role of 3-OHBaP in the developmental toxicity of $\mathrm{BaP}$ follows from the fact that $\mathrm{BaP}$ itself tested negative in the EST without bioactivation (Kamelia et al. 2020). Previous studies have shown that in vivo developmental toxicity can adequately be predicted using reverse dosimetry based on the EST for several compounds, including both compounds for which the developmental toxicity is ascribed to the parent compound itself as well as compounds for which the effect is due to a metabolite ( $\mathrm{Li}$ et al. 2017a; Louisse et al. 2015; Strikwold et al. 2013, 2017). So far, however, this approach has not been assessed for PAHs, although PAH-containing petroleum substances make up a large part of chemicals that require developmental toxicity testing under REACH. The current study shows that the in vitro-in silico approach is suitable for predicting developmental toxicity of $\mathrm{BaP}$ in rats, based on in vitro data of the EST for its major metabolite 3-OHBaP, responsible for the developmental toxicity in vitro.

For reverse dosimetry, EST data for 3-OHBaP and not $\mathrm{BaP}$ were used, as the ES-D3 cells of the EST appear to lack sufficient bioactivation activity to convert $\mathrm{BaP}$ into the active metabolite 3-OHBaP, explaining why $\mathrm{BaP}$ tested negative in the EST (Kamelia et al. 2020). Previous studies confirmed this explanation, since only pre-incubation of $\mathrm{BaP}$ with hamster liver microsomes prior to testing in the EST, resulted in a positive response that reflected the level of 3-OHBaP formation in the pre-incubation (Kamelia et al. 2020). These findings explicate the need for including bioactivation of $\mathrm{BaP}$ to its reactive metabolite $3-\mathrm{OHBaP}$, and further metabolism and clearance of 3-OHBaP in the quantitative in vitro-in vivo extrapolation (QIVIVE) for evaluation of the developmental toxicity of BaP.

The metabolism of $\mathrm{BaP}$ and 3-OHBaP was represented in the current PBK model by the $V_{\max }$ and $K_{\mathrm{m}}$ determined in in vitro incubations with subcellular fractions. Similar studies using rat lung microsomes revealed that pulmonary conversion of $\mathrm{BaP}$ into 3-OHBaP was negligible compared to the conversion in the liver. This finding was supported by previous results from microsomal incubations, showing that the metabolic rate of metabolite formation from $\mathrm{BaP}$ in rat lung was only $0.008 \%$ of liver metabolism (Prough et al. 1979). Further results obtained in the present study for conjugation of 3-OHBaP in incubations with rat liver and lung S9 revealed that also the conjugation of 3-OHBaP mainly occurs in liver. Thus, in the PBK model, formation and clearance of 3-OHBaP was modelled in the liver compartment, while conversion in the lung was considered negligible and not included in the model code.

The PBK model developed was evaluated based on available in vivo data for $3-\mathrm{OHBaP}$ blood concentrations as measured upon dosing $10 \mathrm{mg} \mathrm{BaP} / \mathrm{kg}$ bw by various routes of administration, including intravenous, intratracheal and oral exposure (Marie et al. 2010; Moreau and Bouchard 2015). BaP and 3-OHBaP are highly lipophilic compounds, as reflected by their high $\log P$ value. Evaluation of the BaP PBK model was focused on adequate prediction of 3-OHBaP levels, as $3-\mathrm{OHBaP}$ is the main inducer of developmental toxicity in vitro and the objective of the present study was to translate in vivo EST data for 3-OHBaP into an in vivo dose-response curve for BaP (Kamelia et al. 2020). Comparison of the model predictions to the available in vivo data revealed that the model somewhat overpredicted the blood concentrations of 3-OHBaP reported by Moreau and Bouchard (2015), while it adequately predicted the data reported by Marie et al. (2010) and the scaled Moreau and Bouchard data. Given that both in vivo studies were performed in the same strain of rat, using the same route of administration and a similar $\mathrm{BaP}$ dose level, it appears that the deviations between the model predictions and the reported in vivo data may originate to a substantial extent from variants between the two experimental data sets.

The results from microsomal incubation of BaP reveal that the $V_{\max }$ and catalytic efficiency for formation of 3-OHBaP are 5.7- to 54-fold and 3.3- to 78- folds higher than for formation of the other metabolites. These results indicate that the other metabolic routes play a less prominent role than 3-OHBaP formation in the metabolism of BaP. Furthermore, comparison of the in vitro developmental toxicity in the EST of 3-OHBaP and a mixture of BaP metabolites formed in an incubation of $\mathrm{BaP}$ with hamster liver 
microsomes revealed that the in vitro developmental toxicity of the mixture of BaP metabolites could be fully ascribed to the level of 3-OHBaP in this metabolite mixture (Kamelia et al. 2020). Given these results and considerations, the model was used for translation of the in vitro EST data for 3-OHBaP into a predicted in vivo dose-response curve for developmental toxicity of $\mathrm{BaP}$, using PBK model-facilitated reverse dosimetry. The $\mathrm{ED}_{50}$ values derived from the predicted in vivo dose-response curve for single and repeated oral exposure thus obtained, were in agreement with the $\mathrm{ED}_{50}$ value calculated for the in vivo data of Archibong et al. (2002) and in line with the in vivo data of Bui et al. (1986). These results further support the validity of the PBK model for predicting in vivo $3-\mathrm{OHBaP}$ blood concentrations. The results also indicate that the in vitro-in silico approach provides an adequate estimate of the developmental toxicity of $\mathrm{BaP}$ in rats. In spite of this, it is relevant to note that the EST detects development of embryonic stem cells into beating cardiomyocytes and may not reflect the specific sensitive endpoints of developmental toxicity observed upon BaP exposure in animal studies, such as developmental neurotoxicity ( $\mathrm{Li}$ et al. 2012; McCallister et al. 2008; Sheng et al. 2010; Wormley et al. 2004). Inclusion of in vitro assays representing additional developmental toxicity endpoints may enhance the currently developed approach, broadening its applicability for toxicological risk assessment to an even further extent. It should be acknowledged that the endpoint characterised, the exposure regimen used and the window of sensitivity of the EST protocol may not match the exposure scenario, endpoint characterised and window of sensitivity for a compound when detecting its developmental toxicity in an in vivo study. These discrepancies may be a factor contributing to differences between predicted dose-response curves and observed dose-response data. Furthermore, when translating EST data to in vivo dose-response curves and comparing the predictions thus obtained to in vivo data on developmental toxicity, it is worth to note that in vivo studies may report a variety of endpoints including for example cardiac malformations, resorptions, fetal body weight decrease, skeletal malformation, visceral malformations and also fetal deaths. In previous studies we have shown that the EST in vitro data can be used to adequately predict these different in vivo developmental toxicity endpoints (Kamelia et al. 2017; Li et al. 2016, 2017b; Louisse et al. 2010, 2011, 2015; Strikwold et al. 2012, 2017).

In conclusion, the present study shows that the developed PBK modelling-based reverse dosimetry approach can adequately predict in vivo developmental toxicity of $\mathrm{BaP}$ based on in vitro data from the EST for 3-OHBaP, the metabolite responsible for this adverse effect. The predicted $\mathrm{ED}_{50}$ values adequately reflected the $\mathrm{ED}_{50}$ value calculated from the in vivo data. This study provides a proof of principle for an integrated in vitro-in silico approach for predicting in vivo developmental toxicity of $\mathrm{BaP}$. The method may provide a promising strategy for predicting the developmental toxicity of related polycyclic aromatic hydrocarbons (PAHs), without the need for animal testing.

Supplementary Information The online version contains supplementary material available at https://doi.org/10.1007/s00204-021-03128-7.

Acknowledgements The authors acknowledge Dr. Jerry Campbell and Dr. Harvey Clewell for their contributions to the development of the model code.

Funding This work was supported by Concawe, Belgium (www.conca we.eu) (Grant number: 201506110) and Operationeel Programma Kansen voor West II (EFRO), The Netherlands (KVW-00181).

\section{Declarations}

Conflict of interest Maartje H. Rietdijk, Danlei Wang, and Ivonne M.C.M. Rietjens declare that they have no conflict of interest. Peter J. Boogaard and Lenny Kamelia are employed by Shell International, a member company of Concawe. Both Prof. Boogaard and Dr. Kamelia are totally free (by contract) to freely design and conduct research and express their own scientific opinion without any obligation towards either Shell or Concawe. The current findings are not intended to constitute any product endorsement.

Availability of data and materials Supplementary materials (model code and supplementary figures).

Code availability Model code available in supplementary materials.

Open Access This article is licensed under a Creative Commons Attribution 4.0 International License, which permits use, sharing, adaptation, distribution and reproduction in any medium or format, as long as you give appropriate credit to the original author(s) and the source, provide a link to the Creative Commons licence, and indicate if changes were made. The images or other third party material in this article are included in the article's Creative Commons licence, unless indicated otherwise in a credit line to the material. If material is not included in the article's Creative Commons licence and your intended use is not permitted by statutory regulation or exceeds the permitted use, you will need to obtain permission directly from the copyright holder. To view a copy of this licence, visit http://creativecommons.org/licenses/by/4.0/.

\section{References}

Archibong AE, Inyang F, Ramesh A et al (2002) Alteration of pregnancy related hormones and fetal survival in F-344 rats exposed by inhalation to benzo(a)pyrene. Reprod Toxicol 16(6):801-808. https://doi.org/10.1016/s0890-6238(02)00058-8

Archibong AE, Ramesh A, Inyang F, Niaz MS, Hood DB, Kopsombut $P(2012)$ Endocrine disruptive actions of inhaled benzo(a)pyrene on ovarian function and fetal survival in fisher F-344 adult rats. Reprod Toxicol 34(4):635-643. https://doi.org/10.1016/j.repro tox.2012.09.003

Brown RP, Delp MD, Lindstedt SL, Rhomberg LR, Beliles RP (1997) Physiological parameter values for physiologically based pharmacokinetic models. Toxicol Ind Health 13(4):407-484. https://doi. org/10.1177/074823379701300401 
Buesen R, Genschow E, Slawik B et al (2009) Embryonic stem cell test remastered: comparison between the validated EST and the new molecular FACS-EST for assessing developmental toxicity in vitro. Toxicol Sci 108(2):389-400. https://doi.org/10.1093/ toxsci/kfp012

Bui QQ, Tran MB, West WL (1986) A comparative study of the reproductive effects of methadone and benzo [a] pyrene in the pregnant and pseudopregnant rat. Toxicology 42(2):195-204. https://doi. org/10.1016/0300-483X(86)90009-0

Campbell J, Franzen A, Van Landingham C et al (2016) Predicting lung dosimetry of inhaled particleborne benzo[a]pyrene using physiologically based pharmacokinetic modeling. Inhal Toxicol 28(11):520-535. https://doi.org/10.1080/08958378.2016.1214768

Chou MW (1983) In vitro metabolism of 8- and 9-fluorobenzo[a]pyrene. In: CMDA J (ed) Polynucl Aromat Hydrocarbons Int Symp, 7th. Battelle Press, Columbus

Cohen GM (1990) Pulmonary metabolism of foreign compounds: its role in metabolic activation. Environ Health Perspect 85:31-41. https://doi.org/10.1289/ehp.85-1568313

Cohen GM, Moore BP (1976) Metabolism of (3H)benzo(a)pyrene by different portions of the respiratory tract. Biochem Pharmacol 25(14):1623-1629. https://doi.org/10.1016/0006-2952(76) 90474-3

Crowell SR, Amin SG, Anderson KA et al (2011) Preliminary physiologically based pharmacokinetic models for benzo[a]pyrene and dibenzo[def, p]chrysene in rodents. Toxicol Appl Pharmacol 257(3):365-376. https://doi.org/10.1016/j.taap.2011.09.020

Evans MV, Andersen ME (2000) Sensitivity analysis of a physiological model for 2,3,7,8-tetrachlorodibenzo-p-dioxin (TCDD): assessing the impact of specific model parameters on sequestration in liver and fat in the rat. Toxicol Sci 54(1):71-80. https://doi.org/ 10.1093/toxsci/54.1.71

Feuston MH, Mackerer CR (1996) Developmental toxicity of clarified slurry oil, syntower bottoms, and distillate aromatic extract administered as a single oral dose to pregnant rats. J Toxicol Environ Health 49(1):45-66

Feuston MH, Kerstetter SL, Singer EJ, Mehlman MA (1989) Developmental toxicity of clarified slurry oil applied dermally to rats. Toxicol Ind Health 5(3):587-599

Feuston MH, Low LK, Hamilton CE, Mackerer CR (1994) Correlation of systemic and developmental toxicities with chemical component classes of refinery streams. Fundam Appl Toxicol 22(4):622630. https://doi.org/10.1006/faat.1994.1068

Feuston MH, Hamilton CE, Mackerer CR (1996) Systemic and developmental toxicity of dermally applied distillate aromatic extract in rats. Fundam Appl Toxicol 30(2):276-284. https://doi.org/10. 1006/faat.1996.0065

Genschow E, Spielmann H, Scholz G et al (2002) The ECVAM international validation study on in vitro embryotoxicity tests: results of the definitive phase and evaluation of prediction models. European Centre for the Validation of Alternative Methods. Altern Lab Anim 30(2):151-176. https://doi.org/10.1177/02611 9290203000204

Genschow E, Spielmann H, Scholz G et al (2004) Validation of the embryonic stem cell test in the international ECVAM validation study on three in vitro embryotoxicity tests. Altern Lab Anim 32(3):209-244. https://doi.org/10.1177/026119290403200305

Goth-Goldstein R, Holman H-YN, Russell ML (1999) In vitro model for intestinal uptake of benzo(a)pyrene. Berkeley Lab, https:// indoor.lbl.gov/publications/vitro-model-intestinal-uptake

Gulden M, Morchel S, Tahan S, Seibert H (2002) Impact of protein binding on the availability and cytotoxic potency of organochlorine pesticides and chlorophenols in vitro. Toxicology $175(1-$ 3):201-213. https://doi.org/10.1016/s0300-483x(02)00085-9

Hamernik KL (1984) A comparative study of the metabolism of 6-methylbenzo[a]pyrene and benzo[a]pyrene by rat liver microsomes. Uniformed Services University of the Health Sciences

Hamernik KLC, Pei Lu, Chou MW, Fu PP, Yang SK (1983) Metabolic activation of 6-methylbenzo[a]pyrene. In: Cooke MD, Anthony J (eds) Polynucl aromat hydrocarbons int symp, 7th. Battelle Press, Columbus, pp 583-597

Heredia Ortiz R, Maître A, Barbeau D, Lafontaine M, Bouchard M (2014) Use of physiologically-based pharmacokinetic modeling to simulate the profiles of 3-hydroxybenzo(a)pyrene in workers exposed to polycyclic aromatic hydrocarbons. PLoS ONE 9(7):e102570-e102570. https://doi.org/10.1371/journal.pone. 0102570

Heredia-Ortiz R, Bouchard M (2013) Understanding the linked kinetics of benzo(a)pyrene and 3-hydroxybenzo(a)pyrene biomarker of exposure using physiologically-based pharmacokinetic modelling in rats. J Pharmacokinet Pharmacodyn 40(6):669-682. https://doi.org/10.1007/s10928-013-9338-9

Heredia-Ortiz R, Bouchard M, Marie-Desvergne C, Viau C, Maitre A (2011) Modeling of the internal kinetics of benzo(a)pyrene and 3-hydroxybenzo(a)pyrene biomarker from rat data. Toxicol Sci 122(2):275-287. https://doi.org/10.1093/toxsci/kfr135

Hood DB, Nayyar T, Ramesh A, Greenwood M, Inyang F (2000) Modulation in the developmental expression profile of Sp1 subsequent to transplacental exposure of fetal rats to desorbed benzo[a]pyrene following maternal inhalation. Inhal Toxicol 12(6):511-535. https://doi.org/10.1080/089583700402897

Houston JB, Galetin A (2008) Methods for predicting in vivo pharmacokinetics using data from in vitro assays. Curr Drug Metab 9(9):940-951. https://doi.org/10.2174/138920008786485164

Jagt K, Munn S, Torslov J, Bruijn J (2004) Alternative approaches can reduce the use of test animals under REACH. Report EUR 2

Kamelia L, Louisse J, de Haan L, Rietjens I, Boogaard PJ (2017) Prenatal developmental toxicity testing of petroleum substances: application of the mouse embryonic stem cell test (EST) to compare in vitro potencies with potencies observed in vivo. Toxicol in Vitro 44:303-312. https://doi.org/10.1016/j.tiv.2017.07.018

Kamelia L, de Haan L, Spenkelink B et al (2020) The role of metabolism in the developmental toxicity of polycyclic aromatic hydrocarbon-containing extracts of petroleum substances. J Appl Toxicol 40(3):330-341. https://doi.org/10.1002/jat.3906

Koehl W, Amin S, Staretz ME et al (1996) Metabolism of 5-methylchrysene and 6-methylchrysene by human hepatic and pulmonary cytochrome P450 enzymes. Cancer Res 56(2):316-324

Li Z, Chadalapaka G, Ramesh A et al (2012) PAH particles perturb prenatal processes and phenotypes: protection from deficits in object discrimination afforded by dampening of brain oxidoreductase following in utero exposure to inhaled benzo(a)pyrene. Toxicol Sci 125(1):233-247. https://doi.org/10.1093/toxsci/kfr261

Li H, Flick B, Rietjens IM, Louisse J, Schneider S, van Ravenzwaay B (2016) Extended evaluation on the ES-D3 cell differentiation assay combined with the BeWo transport model, to predict relative developmental toxicity of triazole compounds. Arch Toxicol 90(5):1225-1237. https://doi.org/10.1007/s00204-015-1541-6

Li Z, Vervoort R, van Ravenzwaay L (2017a) Use of physiologically based kinetic modeling-facilitated reverse dosimetry of in vitro toxicity data for prediction of in vivo developmental toxicity of tebuconazole in rats. Toxicol Lett 266:85-93. https://doi.org/10. 1016/j.toxlet.2016.11.017

Li H, Zhang M, Vervoort J, Rietjens IM, van Ravenzwaay B, Louisse J (2017b) Use of physiologically based kinetic modeling-facilitated reverse dosimetry of in vitro toxicity data for prediction of in vivo developmental toxicity of tebuconazole in rats. Toxicol Lett 266:85-93. https://doi.org/10.1016/j.toxlet.2016.11.017

Lobell M, Sivarajah V (2003) In silico prediction of aqueous solubility, human plasma protein binding and volume of distribution of 
compounds from calculated $\mathrm{pKa}$ and AlogP98 values. Mol Divers 7(1):69-87. https://doi.org/10.1023/b:modi.0000006562.93049.36

Louisse J, de Jong E, van de Sandt JJ et al (2010) The use of in vitro toxicity data and physiologically based kinetic modeling to predict dose-response curves for in vivo developmental toxicity of glycol ethers in rat and man. Toxicol Sci 118(2):470-484. https://doi.org/ 10.1093/toxsci/kfq270

Louisse J, Gonen S, Rietjens IM, Verwei M (2011) Relative developmental toxicity potencies of retinoids in the embryonic stem cell test compared with their relative potencies in in vivo and two other in vitro assays for developmental toxicity. Toxicol Lett 203(1):1-8. https://doi.org/10.1016/j.toxlet.2011.02.012

Louisse J, Bosgra S, Blaauboer BJ, Rietjens IM, Verwei M (2015) Prediction of in vivo developmental toxicity of all-trans-retinoic acid based on in vitro toxicity data and in silico physiologically based kinetic modeling. Arch Toxicol 89(7):1135-1148. https:// doi.org/10.1007/s00204-014-1289-4

Marie C, Bouchard M, Heredia-Ortiz R, Viau C, Maitre A (2010) A toxicokinetic study to elucidate 3-hydroxybenzo(a)pyrene atypical urinary excretion profile following intravenous injection of benzo(a)pyrene in rats. J Appl Toxicol 30(5):402-410. https:// doi.org/10.1002/jat.1511

McCallister MM, Maguire M, Ramesh A et al (2008) Prenatal exposure to benzo(a)pyrene impairs later-life cortical neuronal function. Neurotoxicology 29(5):846-854. https://doi.org/10.1016/j.neuro. 2008.07.008

Moreau M, Bouchard M (2015) Comparison of the kinetics of various biomarkers of benzo[a]pyrene exposure following different routes of entry in rats. J Appl Toxicol 35(7):781-790. https://doi.org/10. 1002/jat.3070

Moserova M, Kotrbova V, Aimova D, Sulc M, Frei E, Stiborova M (2009) Analysis of benzo[a]pyrene metabolites formed by rat hepatic microsomes using high pressure liquid chromatography: optimization of the method. Interdiscip Toxicol 2(4):239-244. https://doi.org/10.2478/v10102-009-0024-0

Poulin P, Krishnan K (1995) An algorithm for predicting tissue: blood partition coefficients of organic chemicals from n-octanol: water partition coefficient data. J Toxicol Environ Health 46(1):117129. https://doi.org/10.1080/15287399509532021

Poulin P, Theil FP (2000) A priori prediction of tissue:plasma partition coefficients of drugs to facilitate the use of physiologicallybased pharmacokinetic models in drug discovery. J Pharm Sci 89(1):16-35. https://doi.org/10.1002/(sici)1520-6017(200001) 89:1\%3c16::Aid-jps3\%3e3.0.Co;2-e

Prough RA, Patrizi VW, Okita RT, Masters BSS, Jakobsson SW (1979) Characteristics of benzo $(<\mathrm{em}>\mathrm{a}</ \mathrm{em}>)$ pyrene metabolism by kidney, liver, and lung microsomal fractions from rodents and humans. Cancer Res 39(4):1199

Punt A, Pinckaers N, Peijnenburg A, Louisse J (2021) Development of a web-based toolbox to support quantitative in-vitro-to-in-vivo extrapolations (QIVIVE) within nonanimal testing strategies. Chem Res Toxicol 34(2):460-472. https://doi.org/10.1021/acs. chemrestox.0c00307

Ramesh A, Hood DB, Inyang F et al (2002) Comparative metabolism, bioavailability, and toxicokinetics of benzo[a ]pyrene in rats after acute oral, inhalation, and intravenous administration. Polycyclic Aromat Compd 22(3-4):969-980. https://doi.org/10.1080/10406 630290104121

Sheng L, Ding X, Ferguson M et al (2010) Prenatal polycyclic aromatic hydrocarbon exposure leads to behavioral deficits and downregulation of receptor tyrosine kinase, MET. Toxicol Sci 118(2):625634. https://doi.org/10.1093/toxsci/kfq304
Sims P (1968) The synthesis of 8- and 9-hydroxybenzo[a]pyrene and the role of the products in benzo[a]pyrene metabolism. J Chem Soc Perkin 1(1):32-34. https://doi.org/10.1039/j39680000032

Strikwold M, Woutersen RA, Spenkelink B, Punt A, Rietjens IM (2012) Relative embryotoxic potency of p-substituted phenols in the embryonic stem cell test (EST) and comparison to their toxic potency in vivo and in the whole embryo culture (WEC) assay. Toxicol Lett 213(2):235-242. https://doi.org/10.1016/j.toxlet. 2012.07.005

Strikwold M, Spenkelink B, Woutersen RA, Rietjens IM, Punt A (2013) Combining in vitro embryotoxicity data with physiologically based kinetic (PBK) modelling to define in vivo doseresponse curves for developmental toxicity of phenol in rat and human. Arch Toxicol 87(9):1709-1723. https://doi.org/10.1007/ s00204-013-1107-4

Strikwold M, Spenkelink B, de Haan LHJ, Woutersen RA, Punt A, Rietjens I (2017) Integrating in vitro data and physiologically based kinetic (PBK) modelling to assess the in vivo potential developmental toxicity of a series of phenols. Arch Toxicol 91(5):2119-2133. https://doi.org/10.1007/s00204-016-1881-x

Sun D, Lennernas H, Welage LS et al (2002) Comparison of human duodenum and Caco-2 gene expression profiles for 12,000 gene sequences tags and correlation with permeability of 26 drugs. Pharm Res 19(10):1400-1416. https://doi.org/10.1023/a:10204 83911355

Torbert HC (1935) The effect of fasting on the serum protein concentration of the rat: with special reference to the question of the existence of an immediately utilizable circulating protein fraction. J Exp Med 62(1):1-10. https://doi.org/10.1084/jem.62.1.1

Veignie E, Rafin C, Woisel P, Sahraoui ALH, Cazier F (2002) Metabolization of the polycyclic aromatic hydrocarbon benzo(a)pyrene by a non-white rot fungus (Fusarium solani) in a batch reactor. Polycycl Aromat Comp 22(1):87-97. https://doi.org/10.1080/ 10406630210372

Withey JR, Shedden J, Law FC, Abedini S (1993) Distribution of benzo[a]pyrene in pregnant rats following inhalation exposure and a comparison with similar data obtained with pyrene. J Appl Toxicol 13(3):193-202. https://doi.org/10.1002/jat.2550130310

Wormley DD, Chirwa S, Nayyar T et al (2004) Inhaled benzo(a)pyrene impairs long-term potentiation in the $\mathrm{F} 1$ generation rat dentate gyrus. Cell Mol Biol (noisy-Le-Grand) 50(6):715-721

Wu J, Ramesh A, Nayyar T, Hood DB (2003) Assessment of metabolites and AhR and CYP1A1 mRNA expression subsequent to prenatal exposure to inhaled benzo(a)pyrene. Int J Dev Neurosci 21(6):333-346. https://doi.org/10.1016/s0736-5748(03)00073-x

Yang SK, Selkirk JK, Plotkin EV, Gelboin HV (1975) Kinetic analysis of the metabolism of benzo(a)pyrene to phenols, dihydrodiols, and quinones by high-pressure chromatography compared to analysis by aryl hydrocarbon hydroxylase assay, and the effect of enzyme induction. Cancer Res 35(12):3642-3650

Zhang M, van Ravenzwaay B, Fabian E, Rietjens I, Louisse J (2018) Towards a generic physiologically based kinetic model to predict in vivo uterotrophic responses in rats by reverse dosimetry of in vitro estrogenicity data. Arch Toxicol 92(3):1075-1088. https:// doi.org/10.1007/s00204-017-2140-5

Publisher's Note Springer Nature remains neutral with regard to jurisdictional claims in published maps and institutional affiliations. 